\title{
A synergistic study of a CFD and semi-analytical models for aeroelastic analysis of bridges in turbulent wind conditions
}

\author{
Igor Kavrakova,** Guido Morgenthal $^{\mathrm{b}}$ \\ ${ }^{a}$ Research Training Group 1462, Bauhaus-University Weimar, Marienstr. 7, Weimar 99423, Germany \\ ${ }^{b}$ Chair of Modelling and Simulation of Structures, Bauhaus-University Weimar, Marienstr. 13, Weimar 99423, Germany
}

\begin{abstract}
Long-span bridges are prone to wind-induced vibrations. Therefore, a reliable representation of the aerodynamic forces acting on a bridge deck is of a major significance for the design of such structures. This paper presents a systematic study of the two-dimensional (2D) fluid-structure interaction of a bridge deck under smooth and turbulent wind conditions. Aerodynamic forces are modeled by two approaches: a computational fluid dynamics (CFD) model and six semianalytical models. The vortex particle method is utilized for the CFD model and the free-stream turbulence is introduced by seeding vortex particles upstream of the deck with prescribed spectral characteristics. The employed semi-analytical models are based on the quasi-steady and linear unsteady assumptions and aerodynamic coefficients obtained from CFD analyses.

The underlying assumptions of the semi-analytical aerodynamic models are used to interpret the results of buffeting forces and aeroelastic response due to a free-stream turbulence in comparison with the CFD model. Extensive discussions are provided to analyze the effect of linear fluid memory and quasi-steady nonlinearity from a CFD perspective. The outcome of the analyses indicates that the fluid memory is a governing effect in the buffeting forces and aeroelastic response, while the effect of the nonlinearity is overestimated by the quasi-steady models. Finally, flutter analyses are performed and the obtained critical velocities are further compared with wind tunnel results, followed by a brief examination of the post-flutter behavior. The results of this study provide a deeper understanding of the extent of which the applied models are able to replicate the physical processes for fluid-structure interaction phenomena in bridge aerodynamics and aeroelasticity.
\end{abstract}

Keywords: Aerodynamic nonlinearity, Fluid memory, Vortex particle method, Buffeting, Flutter, Long-span bridges

\section{Introduction}

Multiple separation and reattachment points, a high Reynolds number and complex geometries are only a few of many reasons why modeling of the wind-bridge interaction has been an active research field in the last few decades. The process of modeling the aerodynamic forces acting on a bridge deck is a challenging task, which has been traditionally approached by means of semi-analytical models (or reduced mathematical models). This approach finds its origins in the analytical potential-flow theory for airfoils (Fung, 1993; Sears, 1941; Theodorsen, 1949), utilizing the quasi-steady and linear unsteady assumptions. In bridge aerodynamics, the semi-analytical models based on the quasi-steady (Diana et al., 1993; Kovacs et al., 1992) and linear unsteady (Davenport, 1962; Scanlan, 1978) assumptions are complemented by aerodynamic coefficients, which describe the fluid-structure interaction. These aerodynamic coefficients are obtained experimentally or numerically as no analytical solution is presently feasible. As most of the developed semi-analytical models revolve around the quasi-steady and linear unsteady assumptions, it is beneficial to introduce them in a concise manner from a perspective of bridge aerodynamics. For purpose of this study, the quasi-steady assumption is defined as follows (adapted from Fung (1993)): The aerodynamic characteristics of a bridge deck for a time-variable angle of attack at any instant of time are the same as the characteristics for an equivalent constant angle of attack. Correspondingly, the linear unsteady assumption is defined as follows: The aerodynamic characteristics of a bridge deck for a time-variable angle of attack, at any instant of time, are the same as the characteristics considering the evolution of the angle of attack for small amplitudes, where linearity and the superposition principle apply. Since the aerodynamic characteristics are in general nonlinearly dependent on the angle of attack, the semi-analytical aerodynamic models tend to account either for the linear fluid memory (evolution of the angle of attack) or the quasi-steady nonlinearity. With increasing spans and modern shape of bridge decks, the linear unsteady and quasi-steady assumptions are challenged. The dynamic relation between a deck motion and/or incoming gust and the unsteady aerodynamic forces behave nonlinearly for high amplitudes of the angle of attack (Wu and Kareem, 2015; Zhang et al., 2017) and the forces contain higher order harmonics for a

${ }^{*}$ Corresponding author. Tel. +49 (0) 3643584109

Email address: igor.kavrakov@uni-weimar.de (Igor Kavrakov) 
single frequency input (Diana et al., 2008, 2010). Several semi-analytical models have been introduced to account for the nonlinear behavior (Chen and Kareem (2001); Diana et al. (1993, 2010); Wu and Kareem (2015)). However, none of them fully describes the nonlinear unsteady behavior of the forces, including the local non-stationary, vortex shedding and inertial mass effects.

As an alternative to the semi-analytical and experimental models, CFD models have recently gained considerable attention for modeling the wind-bridge interaction. The 2D vortex particle method has been extensively applied to bluffbody aerodynamics problems, specifically in bridge aerodynamics. Its success, besides a very low computational cost even for complex geometries, is due to generally good agreement with wind tunnel test results (Abbas et al., 2017; Farsani et al., 2014; Ge and Xiang, 2008; Larsen and Walther, 1997; Morgenthal and McRobie, 2002; Taylor and Vezza, 2009), which is mainly attributed to the conservation of the angular impulse and energy and insignificant numerical dissipation. The method is based on a discretization of the Navier-Stokes equations by vorticity-carrying particles and when the particle spacing reaches the Kolmogorov dissipation length it becomes a Direct Numerical Simulation (Fukuda and Kamemoto, 2004). In high Reynolds number engineering-type applications the method is used significantly under-resolved and in the common implementations, the main limitations are the two-dimensionality of the flow and lack of turbulence modeling. However, the high accuracy in terms of quantities such as static wind coefficients or flutter derivatives results largely from the robust resolution of flow separation, eddy motion and wake formation (Ferziger, 1993). Recently, a method for modeling free-stream turbulence was introduced by Prendergast (2007), which was applied and validated for the determination of the 2D aerodynamic admittance in Hejlesen et al. (2015); Rasmussen et al. (2010). In this method, inflow particles are introduced upstream of the section, simulating fluctuating velocities with prescribed spectral characteristics. Thus, the main incentives for utilizing this method are the good level of accuracy while retaining computational efficiency and the robust modeling of free-stream turbulence.

This paper studies the similarities and discrepancies between a CFD model, based on the vortex particle method, and six semi-analytical models, based on the linear unsteady and quasi-steady assumptions, in a systematic framework (see Fig. 1). In light of the statements above, the motivation of the study is to analyze the effects of the underlying assumptions implied in the semi-analytical models on the aerodynamic and aeroelastic response from a CFD perspective. The mathematical properties of a CFD model offer a formulation, which is able to replicate certain aerodynamic effects, otherwise intractable by the commonly used semi-analytical models. Herein, the validity and range of application of the quasi-steady and linear unsteady assumptions are emphasized. Outside their range of application, the unsteady nonlinear effects are highlighted. Although there are former works assessing the semi-analytical models (see e.g. Kavrakov and Morgenthal (2017); Wu and Kareem (2013)), the authors are not aware of a study including comprehensive aerodynamic and aeroelastic analyses under smooth and turbulent free-stream, in which a CFD model is taken as a reference. Furthermore, the study contains a verification of an isotropic free-stream turbulence for the vortex particle method compared to previous studies which include only anisotropic free-stream turbulence (Prendergast, 2007; Rasmussen et al., 2010).

The article is organized as follows: First, the semi-analytical models (Sec. 2) and the CFD model (Sec. 3) are briefly reviewed for discussion purposes. The main contribution of this study is given in Sec. 4, which constitutes 2D aerodynamic and aeroelastic analyses of the Great Belt Bridge. Initially, a flow verification is performed in Sec. 4.1 for an isotropic free-stream turbulence. The first indications of the nonlinearities are discussed in Sec. 4.2 based on the aerodynamic coefficients, which are also validated against former studies. Items of interest for the comparative analyses are the buffeting forces (Sec. 4.3), aerostatic response (Sec. 4.4), aeroelastic response (Sec. 4.5), critical flutter velocity and post-flutter behavior (Sec. 4.6). Section 5 constitutes three critical remarks regarding the two-dimensionality of the flow, validity of the numerical model and comparability of the results. Finally, a summary is given in Sec. 6 .

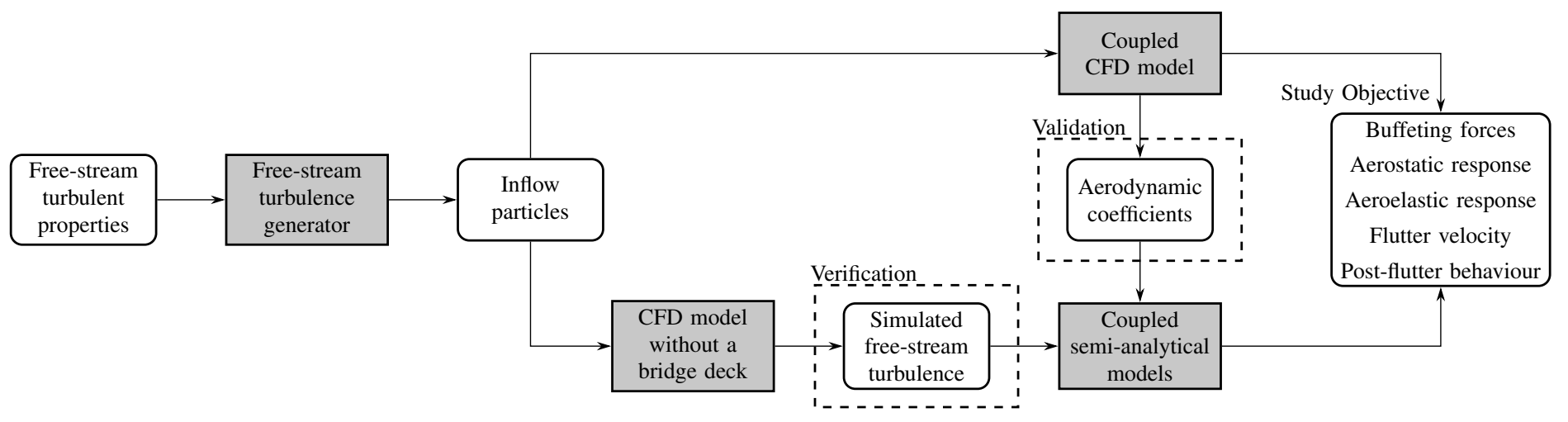

Figure 1: Framework of the synergistic study of a CFD and semi-analytical models for aerodynamic and aeroelastic analyses of bridges. A gray box indicates a model, while a white box indicates an input/output. 


\section{Semi-analytical Models}

In order to formulate the semi-analytical models for the aerodynamic forces, a bridge deck with chord $B$ and depth $H$ is depicted in Fig. 2, describing a wind-structure interaction. The wind velocity is separated into a mean velocity $U$ and fluctuating components $u=u(t)$ and $w=w(t)$ in the horizontal and vertical direction, respectively. The aerodynamic forces acting on the bridge deck due to motion (self-excited), mean wind (static) and free-stream wind fluctuations (buffeting) are the drag force $D$, lift force $L$ and the pitching moment $M$. Considering a system with vertical $h$ and torsional $\alpha$ degrees of freedom, the equations of motion for a dynamic equilibrium are given as follows:

$$
\begin{aligned}
m_{h} \ddot{h}+c_{h} \dot{h}+k_{h} h & =L, \\
m_{\alpha} \ddot{\alpha}+c_{\alpha} \dot{\alpha}+k_{\alpha} \alpha & =M,
\end{aligned}
$$

where $m_{h}, c_{h}$ and $k_{h}$ are the vertical mass, damping and stiffness of the system, respectively, and $m_{\alpha}, c_{\alpha}$ and $k_{\alpha}$ are the rotational mass, damping and stiffness of the system, respectively. The corresponding time derivatives are given in Newton's notation.

Within the quasi-steady assumption, the aerodynamic forces are assumed not to be influenced by the wake. Thus, the aerodynamic forces are obtained by integrating the averaged pressure with respect to the instantaneous angle of attack. The quasi-steady (QS) model for applications in bridge aerodynamics is formulated in the subsequent form (Chen and Kareem, 2002; Kovacs et al., 1992):

$$
L=F_{L} \cos \phi_{L}-F_{D} \sin \phi_{L}, \quad M=F_{M},
$$

for

$$
F_{D}=\frac{1}{2} \rho U_{r D}^{2} B C_{D}\left(\alpha_{e D}\right), \quad F_{L}=-\frac{1}{2} \rho U_{r L}^{2} B C_{L}\left(\alpha_{e L}\right), \quad F_{M}=\frac{1}{2} \rho U_{r M}^{2} B^{2} C_{M}\left(\alpha_{e M}\right),
$$

where $\rho$ is the still air density, $\phi_{j}$ is the dynamic angle of attack, while the drag, lift and moment static wind coefficients are denoted as $C_{D}, C_{L}$ and $C_{M}$, respectively. The static wind coefficients are nonlinearly dependent on the effective angle of attack $\alpha_{e}$, which is obtained as follows:

$$
\alpha_{e j}=\alpha_{s}+\alpha+\phi_{j}=\alpha_{s}+\alpha+\arctan \left(\frac{w+\dot{h}+m_{j} B \dot{\alpha}}{U+u}\right),
$$

where $\alpha_{s}$ is the angle of attack at static equilibrium. The resultant velocity $U_{r j}$ is given as follows:

$$
U_{r j}=\sqrt{(U+u)^{2}+\left(w+\dot{h}+m_{j} B \dot{\alpha}\right)^{2}},
$$

where $m_{j}$ is the aerodynamic center for $j \in\{L, M\}$ in Eqs. $(4,5)$. The term $m_{j} B$ represents a distance between a point, at which the aerodynamic forces are defined (commonly in bridge aerodynamics, the stiffness center), and a point, at which an equivalent (resultant) downwash velocity due to angular motion is positioned. In bridge aerodynamics, the self-excited forces are dependent on the downwash (vertical velocity) at multiple points and on the oscillation frequency (Wu and Kareem, 2013). Hence, an equivalent point is obtained for a selected equivalent quasi-steady state. In other words, the aerodynamic center $m_{j}$ defines a resultant point for each component of the self-excited forces due to rotation. Herein, this parameter is obtained from the flutter derivatives, as defined in the sequel.

Depending on the turbulence intensity and deck oscillations, the instantaneous effective angle of attack can result in large values amounting to $\alpha_{e} \geq 20 \mathrm{deg}$. Since the static wind coefficients are commonly available for a smaller range (e.g. $\pm 10 \mathrm{deg}$ ), they are often linearized. Linearizing Eqs. (2) at the static angle of attack $\alpha_{s}$ and using the small angle approximation, the linear quasi-steady (LQS) model for the aerodynamic forces is obtained as follows (Chen and Kareem, 2002):

$$
\begin{aligned}
& L=-\frac{1}{2} \rho U^{2} B\left[C_{L}+2 C_{L} \frac{u}{U}+\left(C_{L}^{\prime}+C_{D}\right) \frac{w}{U}+\left(C_{L}^{\prime}+C_{D}\right) \frac{\dot{h}+m_{L} B \dot{\alpha}}{U}+C_{L}^{\prime} \alpha\right], \\
& M=\frac{1}{2} \rho U^{2} B^{2}\left[C_{M}+2 C_{M} \frac{u}{U}+C_{M}^{\prime} \frac{w}{U}+C_{M}^{\prime} \frac{\dot{h}+m_{M} B \dot{\alpha}}{U}+C_{M}^{\prime} \alpha\right],
\end{aligned}
$$

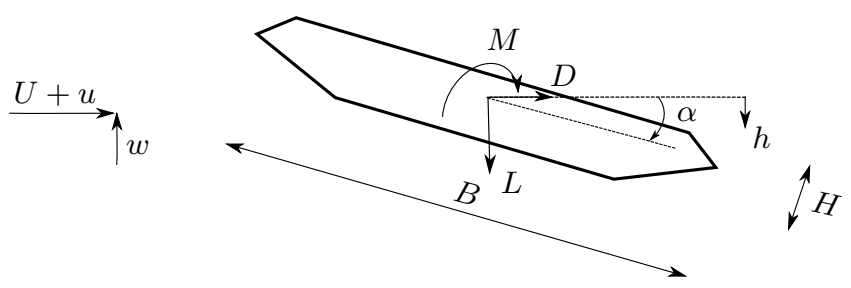

Figure 2: Coordinate system of wind fluctuations and aerodynamic forces acting on a bridge deck cross-section. 
where the $C_{j}=C_{j}\left(\alpha_{s}\right)$ are the static wind coefficients at $\alpha_{s}$ and $C_{j}^{\prime}$ are the derivatives of the static wind coefficients with respect to $\alpha_{s}$ for $j \in\{L, M\}$. The QS and LQS models ignore the unsteady behavior in the buffeting and self-excited forces, often referred to as the fluid memory (or the evolution of the angle of attack).

Unlike to the LQS model, the linear unsteady (LU) model takes into account the unsteadiness of the aerodynamic forces. The fluid memory is included by introducing linear frequency-dependent terms in Eqs. (6), yielding the subsequent relations for the LU model (Davenport, 1962; Jain et al., 1996):

$$
\begin{aligned}
L & =\frac{1}{2} \rho U^{2} B\left[-C_{L}-2 C_{L} \frac{u}{U} \chi_{L u}-\left(C_{L}^{\prime}+C_{D}\right) \frac{w}{U} \chi_{L w}+K H_{1}^{*} \frac{\dot{h}}{U}+K H_{2}^{*} \frac{B \dot{\alpha}}{U}+K^{2} H_{3}^{*} \alpha+K^{2} H_{4}^{*} \frac{h}{B}\right], \\
M & =\frac{1}{2} \rho U^{2} B^{2}\left[C_{M}+2 C_{M} \frac{u}{U} \chi_{M u}+C_{M}^{\prime} \frac{w}{U} \chi_{M w}+K A_{1}^{*} \frac{\dot{h}}{U}+K A_{2}^{*} \frac{B \dot{\alpha}}{U}+K^{2} A_{3}^{*} \alpha+K^{2} A_{4}^{*} \frac{h}{B}\right] .
\end{aligned}
$$

where $\chi_{j w}$ and $\chi_{j u}$ for $j \in\{L, M\}$ are the frequency-dependent aerodynamic admittance functions, accounting for the unsteadiness in the buffeting forces. The aerodynamic admittance is in a complex form $\chi=\chi(K)=F+i G$, dependent on the reduced frequency $K=\omega B / U$, for $i$ being the imaginary number. In Eq. (7), $H_{j}^{*}=H_{j}^{*}(K)$ and $A_{i}^{*}=A_{i}^{*}(K)$ are the flutter derivatives for $j \in\{1,2, \ldots, 4\}$, which account for the fluid memory in the self-excited forces.

Equations (7) are of a mixed nature, that is, they contain frequency- and time-dependent terms. Herein, the transformation of Eqs. (7) in a pure time-domain form is performed by the indicial function approach (Scanlan et al., 1974). In this approach, the aerodynamic admittance and flutter derivatives are approximated by indicial functions, describing the evolution of the aerodynamic forces due to sudden change of the angle of attack. The LU model then yields the subsequent form:

$$
\begin{aligned}
& L=-\frac{1}{2} \rho U^{2} B\left\{C_{L}+2 C_{L} \int_{-\infty}^{s} \Psi_{L u}(s-\tau) \frac{u^{\prime}(\tau)}{U} \mathrm{~d} \tau+\left(C_{L}^{\prime}+C_{D}\right) \int_{-\infty}^{s}\left[\Psi_{L w}(s-\tau) \frac{w^{\prime}(\tau)}{U}+\Phi_{L h}(s-\tau) \frac{h^{\prime \prime}(\tau)}{B}+\Phi_{L \alpha}(s-\tau) \alpha^{\prime}(\tau)\right] \mathrm{d} \tau\right\} \\
& M=\frac{1}{2} \rho U^{2} B^{2}\left\{C_{M}+2 C_{M} \int_{-\infty}^{s} \Psi_{M u}(s-\tau) \frac{u^{\prime}(\tau)}{U} \mathrm{~d} \tau+C_{M}^{\prime} \int_{-\infty}^{s}\left[\Psi_{M w}(s-\tau) \frac{w^{\prime}(\tau)}{U}+\Phi_{M h}(s-\tau) \frac{h^{\prime \prime}(\tau)}{B}+\Phi_{M \alpha}(s-\tau) \alpha^{\prime}(\tau)\right] \mathrm{d} \tau\right\} .
\end{aligned}
$$

In the preceding equations, $s=t U / B$ represents the reduced time as a function of the real time $t$, while $\Psi$ represent the indicial function due to uniform unit-step gust and $\Phi$ is the indicial function due to a unit-step motion. The reduced time derivative is denoted with Lagrange's notation. In this case, the indicial functions are obtained indirectly from the flutter derivatives and aerodynamic admittance functions by means of rational approximation. Further details for the rational approximation can be found in Caracoglia and Jones (2003); Chen and Kareem (2002); Zhang et al. (2011) and are omitted here for the sake of brevity.

The modified quasi-steady (MQS) model is introduced by Øiseth et al. (2010) in order to reduce the ambiguity in the torsional damping related to the aerodynamic center in the original LQS model and to account for the fluid memory in an averaged manner. The idea is based on Eqs. (7), where instead of using a rational approximation, a linear leastsquare fit is performed to approximate the frequency-dependent products by frequency-independent coefficients. Thus, the aerodynamic forces in the MQS model can be expressed as follows:

$$
\begin{aligned}
L & =\frac{1}{2} \rho U^{2} B\left[-C_{L}-2 C_{L} \frac{u}{U}-\left(C_{L}^{\prime}+C_{D}\right) \frac{w}{U}+h_{1} \frac{\dot{h}}{U}+h_{2} \frac{B \dot{\alpha}}{U}+h_{3} \alpha+h_{4} \frac{h}{B}\right], \\
M & =\frac{1}{2} \rho U^{2} B^{2}\left[C_{M}+2 C_{M} \frac{u}{U}+C_{M}^{\prime} \frac{w}{U}+a_{1} \frac{\dot{h}}{U}+a_{2} \frac{B \dot{\alpha}}{U}+a_{3} \alpha+a_{4} \frac{h}{B}\right] .
\end{aligned}
$$

where $h_{j}(1 / K)=H_{j}^{*}(K)$ for $j \in\{1,2\}$ and $h_{j}(1 / K)^{2}=H_{j}^{*}(K)$ for $j \in\{3,4\}$ are the frequency-independent coefficients obtained by least-square fit from the flutter derivatives. The frequency-independent coefficients related to the moment $a_{j}$ are obtained in similar fashion.

Diana et al. (1993) proposed the corrected quasi-steady (CQS) model by modifying the slope of the static wind coefficients in Eqs. (3) with a factor which takes into account an averaged fluid memory. The idea of the CQS model is to retain the nonlinearity considered in the QS model as an advantage over the LU model, while partially modeling the unsteadiness. In the CQS model, the corrected static wind coefficients are formulated as follows:

$$
C_{j}^{*}\left(\alpha_{e j}\right)=C_{j}\left(\alpha_{s}\right)+\int_{\alpha_{s}}^{\alpha_{e j}} K_{j}^{*}(\alpha) C_{j}^{\prime}(\alpha) \mathrm{d} \alpha,
$$

where $K_{j}^{*}$ for $j \in\{D, L, M\}$ represents the frequency-dependent correction coefficient accounting for the averaged fluid memory. In this case, the correction coefficients are obtained from the flutter derivatives, as defined in the sequel.

The concept of the hybrid nonlinear (HNL) model is to utilize the nonlinear QS model for the low reduced frequency range, where the quasi-steady assumption is valid, and the LU model for the high reduced frequency range, where the effect of the fluid memory is significant (Chen and Kareem, 2001). In this manner, the LU model is linearized at the low-frequency effective angle of attack, obtained from the response for the QS model and low-frequency component of the wind fluctuations. The total aerodynamic force then yields as follows:

$$
F_{j}=F_{j}^{Q S}\left(\alpha_{e j}^{l}\right)+\left.F_{j}^{L U}\left(\alpha_{e j}^{h}\right)\right|_{\alpha_{e j}^{l}}
$$


where $F_{j}$ is the total aerodynamic force, while $F_{j}^{Q S}$ (see Eqs. $(2,3)$ ) is the quasi-steady part of the aerodynamic force. The linear unsteady part of the aerodynamic force $F_{j}^{L U}$ (see Eqs. (7)) is a function of the high-frequency effective angle $\alpha_{e j}^{h}$ and is linearized at the low-frequency effective angle $\alpha_{e j}^{l}$ for $j \in\{D, L, M\}$. Diana et al. (2013) modified the low-frequency effective angle of attack for the HNL model by introducing a new lag correction coefficient $n_{j}$ in order to account for the phase lag in the QS model as follows:

$$
\alpha_{e j}^{l}=\alpha_{s}+\alpha^{l}+\arctan \left(\frac{w^{l}+\dot{h}^{l}+m_{j} B \dot{\alpha}^{l}+n_{j} \dot{w}^{l}}{U+u^{l}}\right) .
$$

In this case, the threshold between the low and high reduced frequency range is taken similar to the first natural frequency (Chen and Kareem, 2001). It is noted that the HNL model is not fully nonlinear or fully unsteady; rather a hybrid model based intrinsic understanding of the aerodynamic force.

The aerodynamic center $m$, frequency-dependent correction coefficient $K^{*}$ and lag correction coefficient $n$ are obtained based on the flutter derivatives and aerodynamic admittance at a certain reduced frequency as follows (Diana et al., 1993, 2013):

$$
m_{L}=-\frac{K H_{2}^{*}}{K^{2} H_{3}^{*}+C_{D}}, \quad m_{M}=\frac{K A_{2}^{*}}{K^{2} A_{3}^{*}} ; \quad K_{L}^{*}=-\frac{K H_{3}^{*}}{C_{L}^{\prime}}, \quad K_{M}^{*}=\frac{K A_{3}^{*}}{C_{M}^{\prime}} ; \quad n_{L}=\frac{G_{L w}}{F_{L w}} \frac{B}{K U}, \quad n_{M}=\frac{G_{M w}}{F_{M w}} \frac{B}{K U} .
$$

Since the aerodynamic center and the lag correction coefficients should represent an equivalent quasi-steady state, they are obtained for high reduced velocities $V_{r}=2 \pi / K \geq 15$. The correction coefficients include the averaged fluid memory; hence, the flutter derivatives are interpolated at a reduced velocity corresponding to the central frequency of oscillation. As the correction coefficients are nonlinear, the flutter derivatives are required for varying angles of attack.

In summary, the aerodynamic mechanisms accounted in the semi-analytical models based on their underlying assumptions are as follows: (i) the LQS model is linear and does not consider the fluid memory; (ii) the QS model accounts for the quasi-steady nonlinearity, while neglecting the fluid memory; (iii) the MQS model considers the averaged fluid memory in a linear fashion; (iv) the CQS model includes the averaged fluid memory at the central frequency of oscillation, while retaining the quasi-steady nonlinearity; (v) the LU model takes into account the linear fluid memory and finally, (vi) the HNL model considers the quasi-steady nonlinearity of the low-frequency angle of attack, while neglecting the unsteadiness and takes into account the linear fluid memory of the high-frequency angle of attack.

\section{Computational fluid dynamics model}

A brief description of the governing equations and present numerical implementation of the 2D vortex particle method and the free-stream turbulence is given in this section. The notion is to establish a basis of the CFD model for discussion purposes, while detailed information can be found in the referenced work.

\subsection{Vortex particle method}

For a Newtonian fluid in 2D flow with kinematic viscosity $v$, the Navier-Stokes equations in a vorticity transport formulation are given as follows (Cottet and Koumoutsakos, 2000):

$$
\frac{\partial \omega_{u}}{\partial t}+(\boldsymbol{u} \cdot \nabla) \omega_{u}=v \nabla^{2} \omega_{u},
$$

where $\omega_{u}=\nabla \times \boldsymbol{u}$ is the vorticity and $\boldsymbol{u}$ is the velocity vector. Utilizing the incompressibility condition, the velocity vector can be uniquely described by the curl of the stream function $\psi$ and free-stream velocity vector $\boldsymbol{U}$ as $\boldsymbol{u}=\nabla \times\left(\psi \boldsymbol{e}_{n}\right)+\boldsymbol{U}$, where the unit vector $\boldsymbol{e}_{n}$ is a vector perpendicular to the plane of the velocity field. The relation between the stream function and vorticity then results in a Poisson equation, which is expressed as follows:

$$
\nabla^{2} \psi=-\omega_{u} .
$$

For a domain $D_{f}$, Eq. (15) can be solved using the Green's function, resulting in the Biot-Savart relation, which is given as follows:

$$
\boldsymbol{u}(\boldsymbol{x})=\boldsymbol{U}-\frac{1}{2 \pi} \int_{D_{f}} \frac{(\boldsymbol{x}-\boldsymbol{y}) \times \omega_{u}(\boldsymbol{y})}{|\boldsymbol{x}-\boldsymbol{y}|^{2}} \mathrm{~d} \boldsymbol{y} .
$$

In the vortex particle method, the vorticity field is represented by $N_{p}$ discrete number of particles at location $\boldsymbol{x}_{p}$, where circulation $\Gamma_{p}$ is concentrated. Using the particle representation of the vorticity field, Eq. (16) yields the following:

$$
\boldsymbol{u}\left(\boldsymbol{x}_{p}\right)=\boldsymbol{U}-\frac{1}{2 \pi} \sum_{p=1}^{N_{p}} \frac{\boldsymbol{e}_{n} \times\left(\boldsymbol{x}_{p}-\boldsymbol{x}\right)}{\left|\boldsymbol{x}_{p}-\boldsymbol{x}\right|^{2}} \Gamma_{p}=\boldsymbol{U}-\sum_{p=1}^{N_{p}} \boldsymbol{K}\left(\boldsymbol{x}_{p}-\boldsymbol{x}\right) \Gamma_{p},
$$



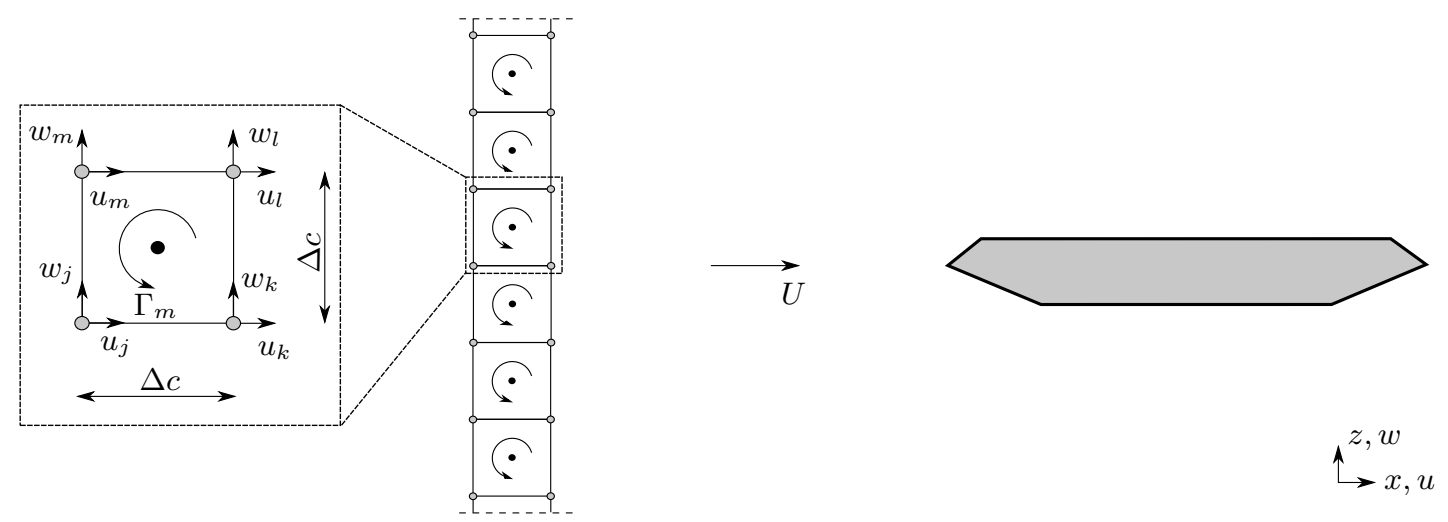

Figure 3: Conversion from velocities, generated on a ladder located upstream of the bridge section, to a particles' with circulation $\Gamma_{m}$. The particles are then released upstream and convected by the mean velocity $U$ simulating free-stream turbulence in the CFD domain.

where $\boldsymbol{K}$ is the velocity kernel. Herein, the vorticity transport equation (Eq. (14)) is solved using the viscous splitting method (Chorin, 1973), in which the convection and diffusion of vortex particles are solved sequentially. Commonly, the kinematics of the particles is obtained by the solution of the Poisson equation (Eq. (15)) on a regularized grid using the Vortex-In-Cell algorithms (Hockney and Eastwood, 1988), utilizing the fast Fourier transform (FFT). In the present implementation, the efficient $\mathrm{P}^{3} \mathrm{M}$ (Morgenthal and Walther, 2007) algorithm is employed. In order to avoid the numerical instabilities caused by the denominator in Eq. (17), the velocity kernel $\boldsymbol{K}$ is replaced by the Gaussian mollified velocity kernel $\boldsymbol{K}_{\epsilon}$ with core radius $\epsilon$. For the solid boundaries, the boundary element method is utilized (Wu, 1976), in which the geometry is discretized on piecewise linear panels. The no-penetration condition is enforced with the solution of a linear system for the strength of the vortex sheets on the boundaries. A unique solution of the linear system of equations for the boundary conditions is achieved by introducing Kelvin's circulation theorem as an additional constraint, that is, $\sum \Gamma=0$. The aerodynamic forces acting on the body are then computed by integration of the pressure.

The stochastic random walk method is utilized herein for the diffusion step, which was introduced by Chorin (1973). In the stochastic random walk method, the particles are displaced after the convection step in an arbitrary direction with a random magnitude with zero mean and a variance of $2 v \Delta t$, where $\Delta t$ is the simulation time-step. The validated platform VXflow is used as a solver and for further details on the numerical implementation and GPU acceleration, the reader is referred to Morgenthal (2002); Morgenthal et al. (2014); Morgenthal and Walther (2007).

\subsection{Synthetic Free-stream Turbulence}

For the simulation of the free-stream turbulent fluctuations, the two-step procedure introduced by Prendergast (2007) is utilized. The first step involves generation of velocities on the corners of a ladder positioned upstream of the section (see Fig. 3). The grid velocities are represented as correlated, stationary random processes with prescribed spectral characteristics. For the digital simulation of the grid velocities, the spectral representation method is employed with the efficient algorithm introduced by Ding et al. (2011). Since the generation of grid velocities is a standard procedure, the details are omitted herein for the sake of brevity and further information can be found in Deodatis (1996); Ding et al. (2011); Shinozuka and Deodatis (1996).

In the second step of the procedure, the grid velocities are converted into concentrated circulation at the center of each cell, yielding a band of vortices positioned upstream of the section. For a square cell with a side length $\Delta c$, the approximate circulation $\Gamma_{a}$ is obtained by numerical integration of the velocity over the cell boundaries at every time-step, utilizing the trapezoidal rule. The modeled strength of each particle $\Gamma_{m}$ is then obtained by modifying $\Gamma_{a}$ as follows (Prendergast, 2007; Prendergast and McRobie, 2006):

$$
\Gamma_{m}=\beta \frac{\pi}{2} \Gamma_{a}=\beta \frac{\pi \Delta c}{4}\left[\left(w_{k}+w_{l}-w_{j}-w_{m}\right)-\left(u_{m}+u_{l}-u_{j}-u_{k}\right)\right],
$$

where the factor $\pi / 2$ is added to account for the difference between the linear velocity approximation and the actual nonlinear velocity distribution of a point vortex. The factor $\pi / 2$ is reduced for a band of vortices due to the "smudging" of circulation (Prendergast, 2007), thus the coefficient $\beta$ is introduced to accommodate for these differences. For isotropic turbulence $\beta \pi / 2 \rightarrow 1$. The particles are injected into the domain at every $\Delta p$ simulation step. Utilizing Taylor's hypothesis of frozen turbulence, the side length of the cell is obtained as $\Delta c=U \Delta p \Delta t$. Since additional circulation is introduced as the injected particles are convected, the additional constraint in the boundary conditions, that is, Kelvin's circulation theorem, needs to be modified as $\sum \Gamma=\sum \Gamma_{m}$. A brief discussion is followed herein on the two-dimensionality of the flow and its impact on the structural response in Sec. 5. 


\section{Aerodynamic and Aeroelastic Analyses}

In this section, several components of the fluid-structure interaction are studied in a step-by-step manner, in order to unveil the different mechanisms contributing to the dynamic response. The reference deck section is from the Great Belt East bridge, which is a $31 \mathrm{~m}$ wide and $4.4 \mathrm{~m}$ deep steel box girder. This section is selected as it is investigated in numerous numerical studies and it may be considered as a standard example for the representation of the aerodynamic behavior of streamlined decks. Figure 2 depicts the shape of the deck, while detailed dimensions are described in Reinhold et al. (1992) for the H9.1 section. It is noted that the railings and crash barriers are not included in the CFD analyses. The mass of the girder is taken as $m_{h}=22.74 \mathrm{t} / \mathrm{m}$, while the mass moment of inertia as $m_{\alpha}=2.47 \times 10^{3} \mathrm{tm}^{2} / \mathrm{m}(\mathrm{Larsen}, 1993)$. The first lateral, vertical and torsional frequencies are set as $f_{p}=0.052 \mathrm{~Hz}, f_{h}=0.100 \mathrm{~Hz}$ and $f_{\alpha}=0.278 \mathrm{~Hz}$, respectively, while the structural damping ratio is chosen as $0.5 \%$.

\subsection{Free-stream turbulence verification}

Before subjecting the structure to a turbulent free-stream, a flow verification is performed without a section in the domain. Substantial parametric studies and investigation on the free-stream turbulence statistics for anisotropic turbulence have been preformed in Hejlesen et al. (2015); Prendergast (2007); Prendergast and McRobie (2006); Rasmussen et al. (2010). Herein, the statistical properties for an isotropic free-stream turbulence are studied. Velocities ranging from 20 to $60 \mathrm{~m} / \mathrm{s}$ with an increment of $10 \mathrm{~m} / \mathrm{s}$ are used for the aeroelastic analyses, for two turbulent cases with turbulence intensity $I$ of $6 \%$ and $10 \%$. The von Kármán power spectral density (PSD) function is utilized, which yields the horizontal $S_{u u}$ and vertical $S_{w w}$ spectral components as follows (Solari and Piccardo, 2000):

$$
\frac{f S_{u u}(f)}{\sigma_{u}^{2}}=\frac{4 f L_{u} / U}{\left[1+70.8\left(f L_{u} / U\right)^{2}\right]^{5 / 6}}, \quad \frac{f S_{w w}(f)}{\sigma_{w}^{2}}=\frac{4 f L_{w} / U\left[1+755.2\left(f L_{w} / U\right)^{2}\right]}{\left[1+283.2\left(f L_{w} / U\right)^{2}\right]^{11 / 6}},
$$

where $f$ is the gust frequency, $\sigma_{j}$ is the standard deviation of the fluctuations and $L_{j}$ is the turbulent length scale for $j \in\{u, w\}$. The longitudinal and vertical length scales are set as $L_{u}=54 \mathrm{~m}$ and $L_{w}=L_{u} / 2=27 \mathrm{~m}$, respectively. In order to retain the second order statistics of the velocity tensor, for isotropic turbulence the cross-spectral component of the velocity fluctuations is set as $S_{u w}=S_{w u}=0$ (Mann, 1994). The horizontal and vertical coherence coefficients are taken as $C_{x}=3$ and $C_{z}=10$, respectively, for Davenport's coherence function. Strictly speaking under Taylor's hypothesis for inviscid flow, the longitudinal coherence coefficient should amount to $C_{x}=0$ (Kristensen, 1979). However, this is not the case herein, in order to partially account for eddy decay and maintain consistency with former studies (Prendergast, 2007; Rasmussen et al., 2010).

The non-dimensional time-step is set as $\Delta s=0.0165$, while the run-time for all analyses is set as $t=700 \mathrm{~s}$, in order to preserve the ratio between the lowest generated wind frequency in correspondence to the structural natural frequency. The turbulent Reynolds number, amounting to $\mathrm{Re}_{L u}=U L_{u} / v=1.8 \times 10^{5}$ (Simiu and Scanlan, 1996), is retained as constant by varying the kinematic viscosity $v$, in order to preserve the turbulent properties of the flow for the chosen wind velocity range. In this way, a comparable basis between the semi-analytical and the CFD model is maintained, since the aerodynamic admittance is obtained under one specific set of turbulent properties. The height of the particle release band is selected as $H_{p}=16 B$, while the particle release factor is taken as $\Delta p=4$ and the coefficient $\beta$ (Eq. (18)) amounts to 0.7. For easier discussion, the velocity sampled on the ladder prior to the conversion into particles' circulation will be referred to as "generated" velocity (see Fig. 3), while velocity tracked within the domain at $(0,0)$ as "simulated" velocity (see Fig. 4). The prescribed statistics are indicated as "target".

Initially, a trial run is performed at $U=30 \mathrm{~m} / \mathrm{s}$ and $I=6 \%$ with a wide trial-domain $(90 B \times 90 B)$ in order to investigate the spatial behavior of the turbulence energy along the center line of the domain $(z / B=0)$. In Fig. 4 , three different regions
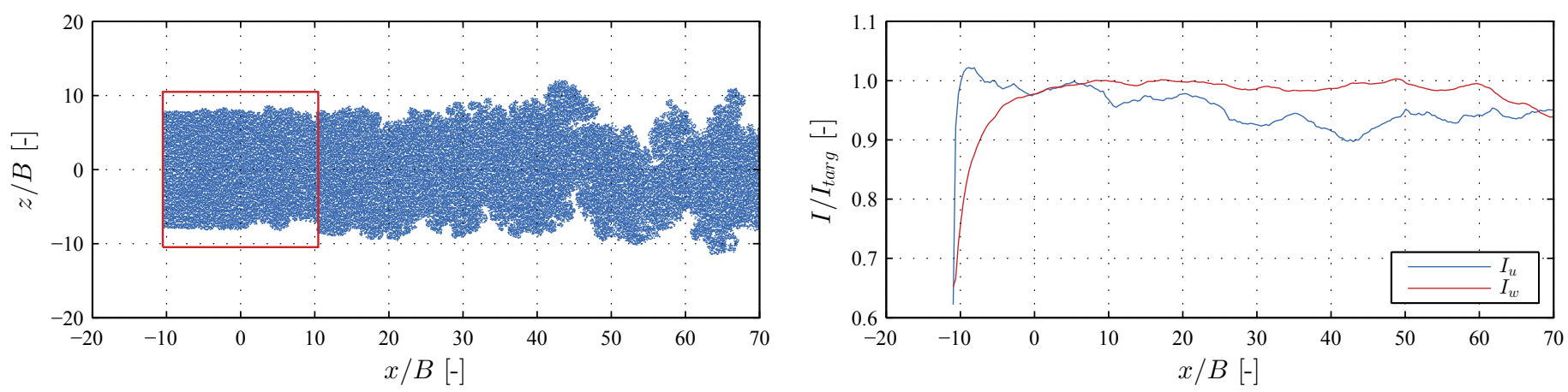

Figure 4: Sample particle map of a large trial domain $90 B \times 90 B$ without a section (left). The red box indicates the selected domain for the analyses. Normalized turbulence intensity $I / I_{\text {targ }}$ along the center line at $z / B=0$ (right) at $U=30 \mathrm{~m} / \mathrm{s}$ and $I_{\text {targ }}=6 \%$. 

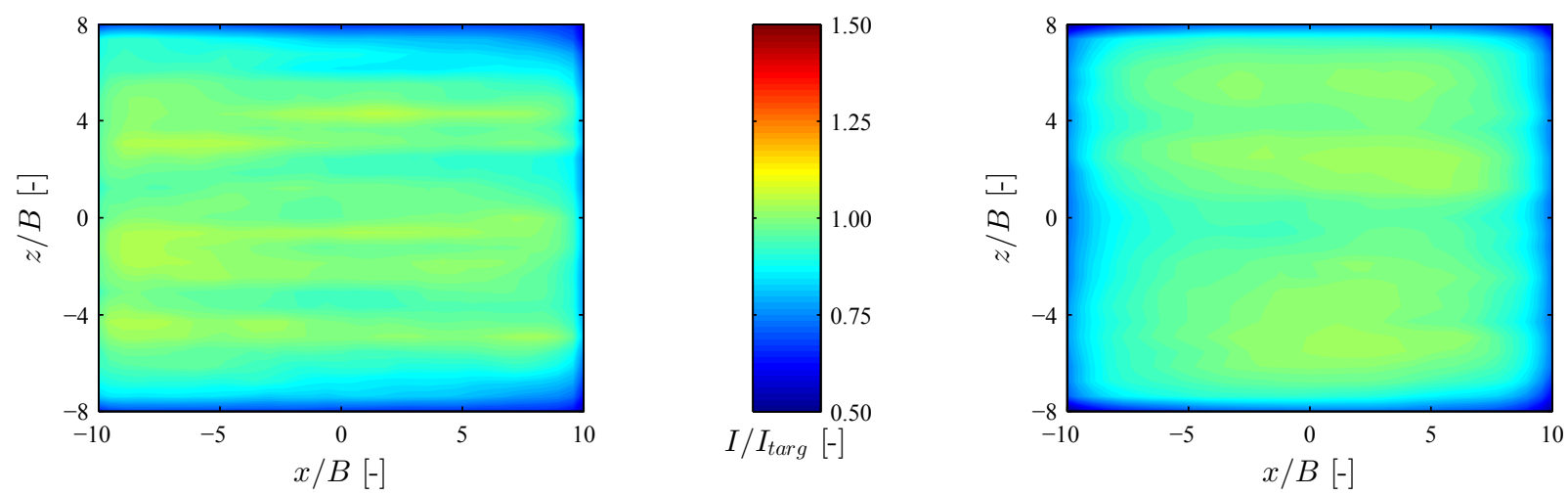

Figure 5: Spatial distribution of the normalized turbulence intensity of the horizontal $I_{u} / I_{\text {targ }}$ (left) and vertical $I_{w} / I_{\text {targ }}$ (right) fluctuations for the selected domain at $U=30 \mathrm{~m} / \mathrm{s}$ and $I_{\text {targ }}=6 \%$.

\begin{tabular}{lrrrrrr}
\hline Parameter & $I_{u}[\%]$ & $I_{w}[\%]$ & $L_{u x}[\mathrm{~m}]$ & $L_{u t}[\mathrm{~m}]$ & $L_{w x}[\mathrm{~m}]$ & $L_{w t}[\mathrm{~m}]$ \\
\hline Target & 6.0 & 6.0 & 54.0 & 54.0 & 27.0 & 27.0 \\
Generated & 6.0 & 6.1 & 52.2 & $/$ & 30.7 & $/$ \\
Simulated & 5.9 & 5.8 & 52.5 & 52.3 & 34.0 & 34.8 \\
\hline
\end{tabular}

Table 1: Verification of turbulence intensities $I$ and length scales $L$ of the generated and simulated wind velocity signals. A "generated" signal is a velocity signal at a node of the ladder before conversion into particles (see Fig. 3), while a "simulated" signal is tracked down velocity at $(0,0)$ in the CFD domain (see Fig. 4). The prescribed free-stream turbulence statistics are denoted as "target". The temporal length scale is obtained as $L_{t}=U / \sigma^{2} \int_{0}^{\infty} R_{t}(\tau) \mathrm{d} \tau$ and the spatial one as $L_{x}=1 / \sigma^{2} \int_{0}^{\infty} R_{x}(x) \mathrm{d} x$.
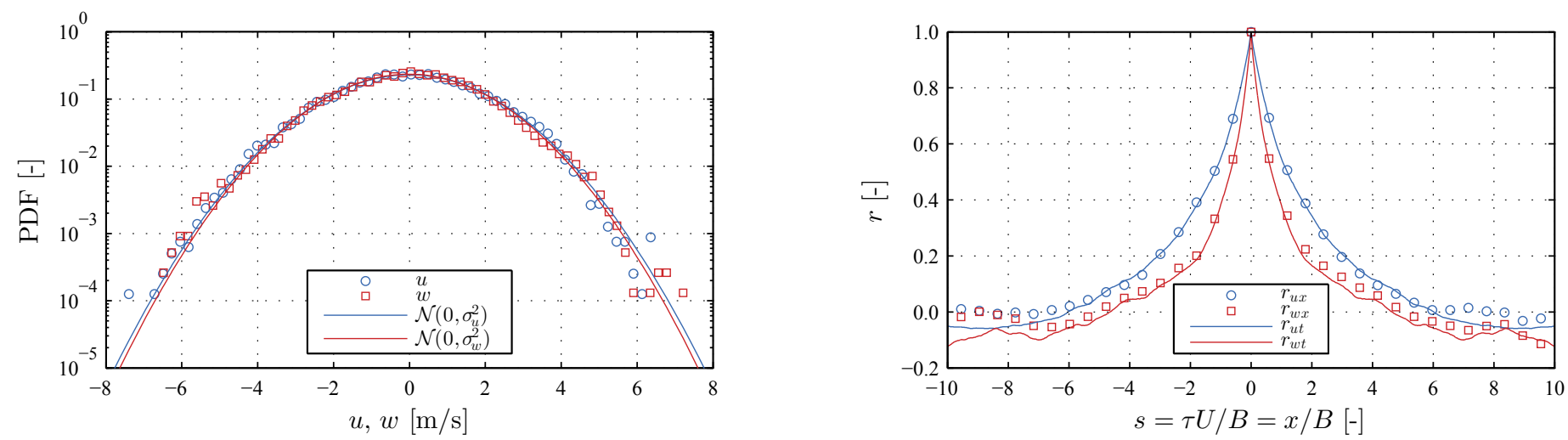

Figure 6: PDF of the turbulent fluctuations and fitted normal distribution $\mathcal{N}$ (left) at $U=30 \mathrm{~m} / \mathrm{s}$ and $I=6 \%$. Spatial and temporal correlation coefficients of the turbulent fluctuations.

of the flow are distinguished based on the instantaneous particle map and the turbulence intensity along the centerline, namely: (i) convergent region - where the kinetic energy converges; (ii) convection region - in which the particles are convected without major interaction and (iii) mixing free flow region - where there is mixing of rotational and free flow domain. It is observed that the longitudinal turbulence energy peaks at $x / B \approx-9$, followed by a constant decay. The vertical turbulence intensity peaks further downstream at $x / B \approx 10$ and remains relatively constant up to $x / B \approx 50$, after which it starts to reduce slowly due to the free-space boundary condition. Similar observations are noted by Rasmussen et al. (2010), who reported that the reduction of vertical turbulent energy starts to decay at approximately at $3.5 H_{p}$. To accommodate a uniform turbulence field and sufficiently developed wake, $10 B$ is chosen as a reasonable distance upstream of the leading edge and behind the trailing edge. The selected domain for a section positioned at $(0,0)$ is marked by the red box on the particle map in Fig. 4. Across the selected domain, uniform turbulence intensity is noted in the area of expected motion and wake of the section (see Fig. 5).

Table 1 gives a comparison between the target, generated and simulated turbulent properties at $(0,0)$ in the selected CFD domain. The turbulence intensities for the simulated velocity yield good correspondence with the target ones. The length scale at the center point is obtained as a function of time as $L_{t}=U / \sigma^{2} \int_{0}^{\infty} R_{t}(\tau) \mathrm{d} \tau$, and as a function of the spatial coordinate $x$ as $L_{x}=1 / \sigma^{2} \int_{0}^{\infty} R_{x}(x) \mathrm{d} x$, where $R_{t}(\tau)$ and $R_{x}(x)$ are the temporal and spatial covariance functions, 

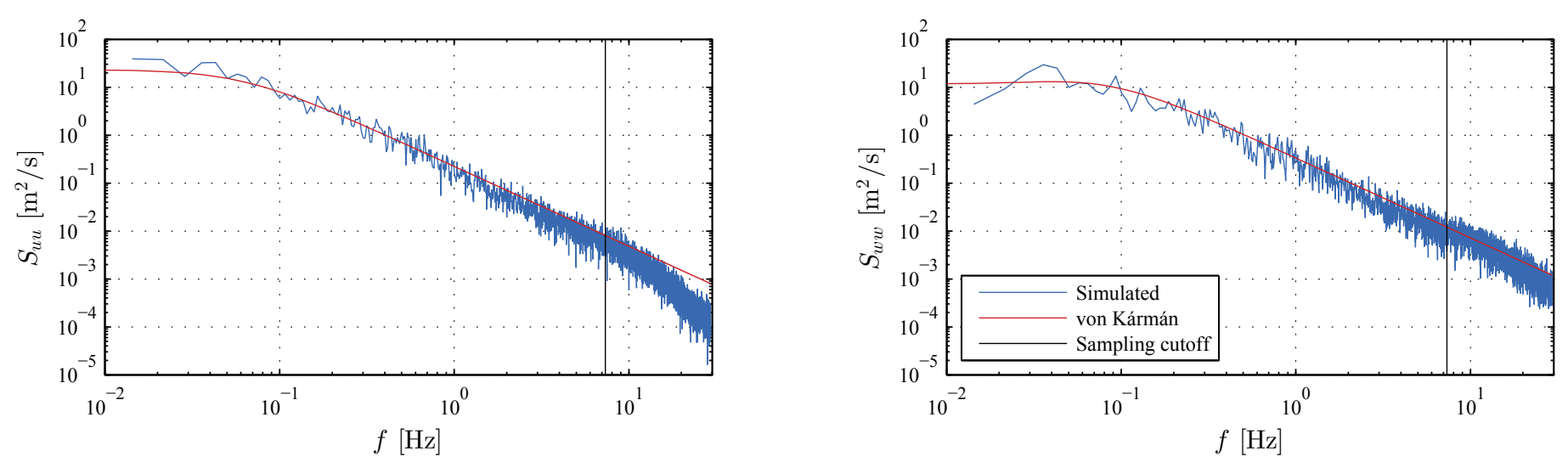

Figure 7: PSD for the horizontal $S_{u u}$ (left) and vertical $S_{w w}$ (right) simulated fluctuating component at $(0,0)$ at $U=30 \mathrm{~m} / \mathrm{s}$ and $I=6 \%$. The sampling cutoff represents a Nyquist sampling rate $f_{\text {gen,max }}$ is for particle release factor $\Delta p=4$.

respectively. The generated and simulated longitudinal length scales correspond good to the target one, while minor overestimation is noted for the vertical ones. In this case, Taylor's hypothesis is generally valid, since there is a good correspondence for the two formulations $\left(L_{t} \approx L_{x}\right)$. The validity of this hypothesis can also be quantitatively evaluated from Fig. 6 (right), where the coefficient of correlation $r$ (or normalized covariance) is depicted with respect to the non-dimensional time. Furthermore, the probability density function (PDF) of the fluctuations is given in Fig. 6 (left). It is observed that both fluctuating components generally follow the normal probability distribution $\mathcal{N}$. However, the Kolmogorov-Smirnov test for normality rejected the null hypothesis at 5\% significance level, which may be attributed to three factors, namely: (i) the conversion from grid velocities to circulation, (ii) artificial energy above the cutoff frequencies due to the injection step $\Delta p$ and (iii) due to the non-Gaussianity of 2D turbulence (Boffeta and Ecke, 2012). Further investigations are required to support the latter statement as the current numerical resolution does not resolve all turbulent scales and no closure model is implemented. The PDF of the wind fluctuations are important for the buffeting forces, and are further discussed in Sec. 4.3.

The PSDs of the simulated wind velocities are compared to the von Kármán PSDs (Eqs. (19)) in Fig. 7. The cutoff frequency amounts to $f_{\text {gen,max }}=7.34 \mathrm{~Hz}$, which corresponds to the Nyquist sampling rate of the generated velocity for $\Delta p=4$ and $U=30 \mathrm{~m} / \mathrm{s}$. Below the sampling frequency, the PSDs of the simulated wind velocities correspond well with the target ones for both velocity components. For frequencies higher than the cutoff frequency, the longitudinal spectral energy decreases while the vertical spectral energy increases. The generated velocity field on the ladder is not divergencefree, as posed by the continuity equation in the Navier-Stokes equations. By converting the grid velocities into circulation by Eq. (18), the divergence-free condition is imposed, which can result in loss of turbulent kinetic energy. This is noted for the case with anisotropic turbulence in Hejlesen et al. (2015); Prendergast (2007); Rasmussen et al. (2010). Nevertheless, the results obtained herein suggest that the loss of turbulent kinetic energy is minor for frequencies lower than the cutoff frequency in the case of isotropic turbulence.

\subsection{Aerodynamic Coefficients}

The aerodynamic coefficients represent the link between the numerical and semi-analytical models. By studying the distinctive features of the aerodynamic coefficients, the nonlinear effects and fluid memory can be preliminarily identified to a certain extent. Moreover, with the comparison of the present results with the aerodynamic coefficients from former

\begin{tabular}{lcrrr}
\hline & $\mathrm{Re}$ & \multicolumn{1}{c}{$C_{D}$} & \multicolumn{1}{c}{$C_{L}$} & \multicolumn{1}{c}{$\mathrm{St}$} \\
\hline Present (VPM) & $1.0 \times 10^{5}$ & 0.060 & 0.065 & $0.106-0.173$ \\
Farsani et al. (2014) (VPM) & $1.0 \times 10^{5}$ & 0.071 & 0.053 & 0.084 \\
Hejlesen et al. (2015) (VPM) & $1.0 \times 10^{4}$ & 0.06 & 0.07 & 0.20 \\
Larsen and Walther (1997) (VPM) & $1.0 \times 10^{5}$ & 0.061 & 0.000 & $0.100-0.168$ \\
Bruno and Khris (2003) (FVM) & $1.0 \times 10^{5}$ & 0.071 & -0.195 & $0.124-0.164$ \\
Lee et al. (2016) (FVM) & $1.0 \times 10^{5}$ & 0.05 & 0.03 & 0.160 \\
Frandsen (2004) (FEM) & $6.2 \times 10^{6}$ & 0.072 & -0.08 & 0.26 \\
Reinhold et al. (1992) (EXP) & $1.0 \times 10^{5}$ & 0.080 & 0.010 & $0.109-0.158$ \\
\hline
\end{tabular}

Table 2: Comparison of static wind coefficients $C_{D}, C_{L}$ and Strouhal number St at $\alpha_{s}=0$ deg with experimental (EXP) results and former numerical studies based on the vortex particle method (VPM), finite volume method (FVM) and finite element method (FEM). 

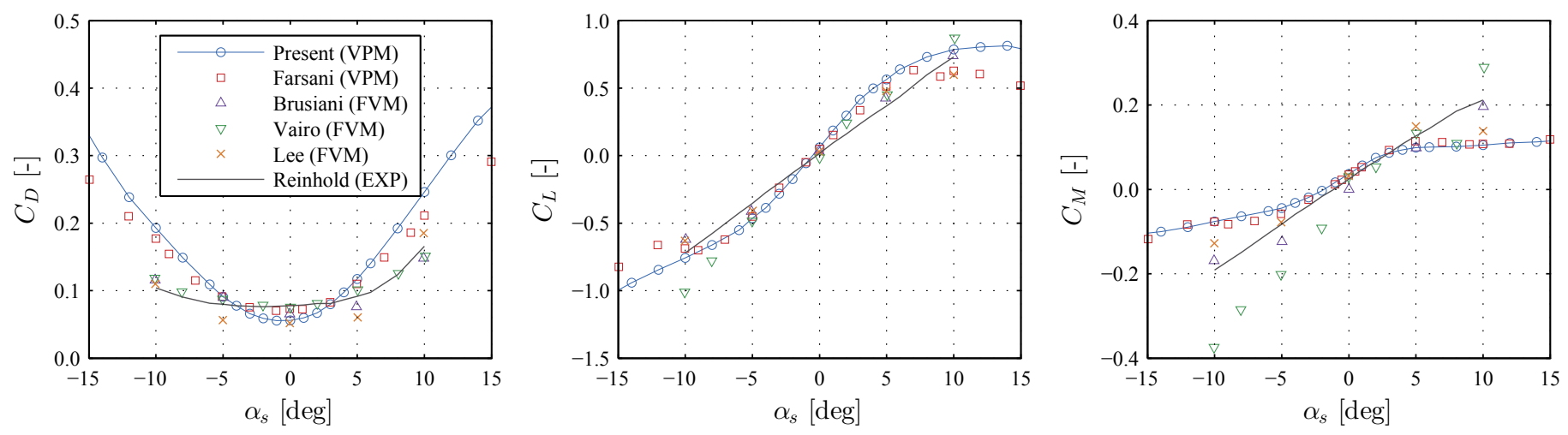

Figure 8: Static wind coefficients compared to former studies (Brusiani et al., 2013; Farsani et al., 2014; Lee et al., 2016; Reinhold et al., 1992; Vairo, 2003): $\operatorname{drag} C_{D}$ (left), lift $C_{L}$ (center) and moment $C_{M}$ (right). The abbreviations in the legend are given in the caption of Tab. 2.

numerical and experimental studies, a sense of the validity of the numerical simulations is acquired. The section is discretized into 250 panels with an average length amounting to $\Delta l / B=8.06 \times 10^{-3}$. The core radius $\epsilon$ for the Gaussian kernel (see Sec. 3.1) is computed from the non-dimensional relation as $\epsilon / \Delta l=1.2$, which satisfies the criterion $4 \sqrt{2} \epsilon / \Delta c \lesssim$ 1 given by Prendergast (2007) in order to avoid excessive smoothing of the free-stream turbulence, and thus, loss of turbulent energy. In this case, this criterion amounts to $4 \sqrt{2} \epsilon / \Delta c=0.87$. The additional equipment (barriers, fairings, railings, etc.) is not modeled in this case, in order to avoid the ambiguity in the porosity by the vortex particle method.

The static wind coefficients are determined for smooth free-stream and each simulation is performed for a time amounting to $s=450$ chords, of which 400 chords are used for the averaging. Table 2 summarizes the mean values of the static wind coefficients and Strouhal number $\mathrm{St}=f_{s} H / U$ at $\alpha_{s}=0 \mathrm{deg}$, where $f_{s}$ is the vortex shedding frequency. Since there is not a clear peak in the spectral amplitude of the lift coefficient, the Strouhal number is computed as a range rather than a value. A similar observation is noted by Kuroda (1997) for shallow box sections, while (Farsani et al., 2014) noted broadband frequency spectrum of the lift for the Great Belt Bridge section. Figure 8 depicts the static wind coefficients with respect to the static angle of attack. It is evident that the value of the drag coefficient is slightly reduced compared to the experimental results, and a change of the slope is indicated in the moment coefficient at $\alpha_{s} \approx \pm 5$ deg. As it can be seen from the figure, some of these features appear in other numerical studies as well. The ratio of the experimental model used for the static wind coefficients, section H4.1 (Reinhold et al., 1992), amounts to $B / H=6.81$. This ratio is lower than the one for the section $\mathrm{H} 9.1(B / H=7.05)$, which is used herein. An increased $B / H$ ratio, insufficient level of detailing of the deck in the numerical model and the presence of free-stream turbulence $\left(I_{u}=7.5 \%\right)$ in the experimental tests could partially explain the scattering in the static wind coefficients for the numerical studies. The increased surface due to the auxiliary equipment gathers additional drag force, and thus, the mean drag coefficient is larger for the experimental values, as noted by Larsen and Walther (1997). Bruno and Khris (2003) note that the side barriers influence the reattachment point and prevent formation of larger bubble on the upper side, while the blockage effect of the railings contributes to higher suction on the lower surface. Both of these effects influence the lift coefficient compared to a bare section, partially explaining the higher slope of the lift coefficient in some of the numerical studies. From the trend of the lift and moment coefficients, it can be observed that the linear assumption is valid in $\approx \pm 5$ deg interval for the static wind coefficients.

The flutter derivatives are determined for smooth free-stream and angle of attack ranging from -4 deg to 4 deg with an increment of $2 \mathrm{deg}$. Utilizing the forced oscillation method, a minimum of 10 cycles at each reduced velocity are performed for the computation. The amplitude of oscillation for the torsional motion is set to $1 \mathrm{deg}$, while the amplitude of the vertical motion was varied such that the $\operatorname{angle} \arctan (\dot{h} / U)$ remains also $1 \mathrm{deg}$. At $\alpha_{s}=0 \mathrm{deg}$, good correspondence is observed between the obtained flutter derivatives and experimental results (see Fig. 9). Furthermore, the indicial functions, obtained from the flutter derivatives, are given in the same figure. Although there is an abrupt change in the first few steps due to the temporal singularity at $V_{r} \rightarrow 0$, the indicial functions are smooth with relatively short rise-time, as expected for a streamlined bridge deck. It is noted that, determining the flutter derivatives under smooth free-stream for aeroelastic analyses with turbulent free-stream does not account for the effect of the free-stream turbulence on the self-excited forces. Haan and Kareem (2016) showed that both turbulence intensity and length-scales impact the flutter derivatives. However, a standard procedure for separation of the buffeting from self-excited forces is not well-established. This warrants further investigation including experimental validation.

In Fig. 10, the flutter derivatives for varying angles of attack are depicted. A higher sensitivity of the flutter derivatives is observed for the positive angles than for negative angles. For $\alpha_{s}=4 \mathrm{deg}$, the sign of $A_{2}^{*}$ changes to positive for reduced velocities above $V_{r}=6$, which indicates torsional flutter. Significantly longer rise-time and higher overshooting amplitudes are noted for the indicial functions at $\alpha_{s}= \pm 4 \mathrm{deg}$. Since the aerodynamic nonlinearity outside the interval of $\alpha_{s}= \pm 4 \mathrm{deg}$ is evident in the static wind coefficients (see Fig. 8), a linearization of the self-excited forces in this region might result in 

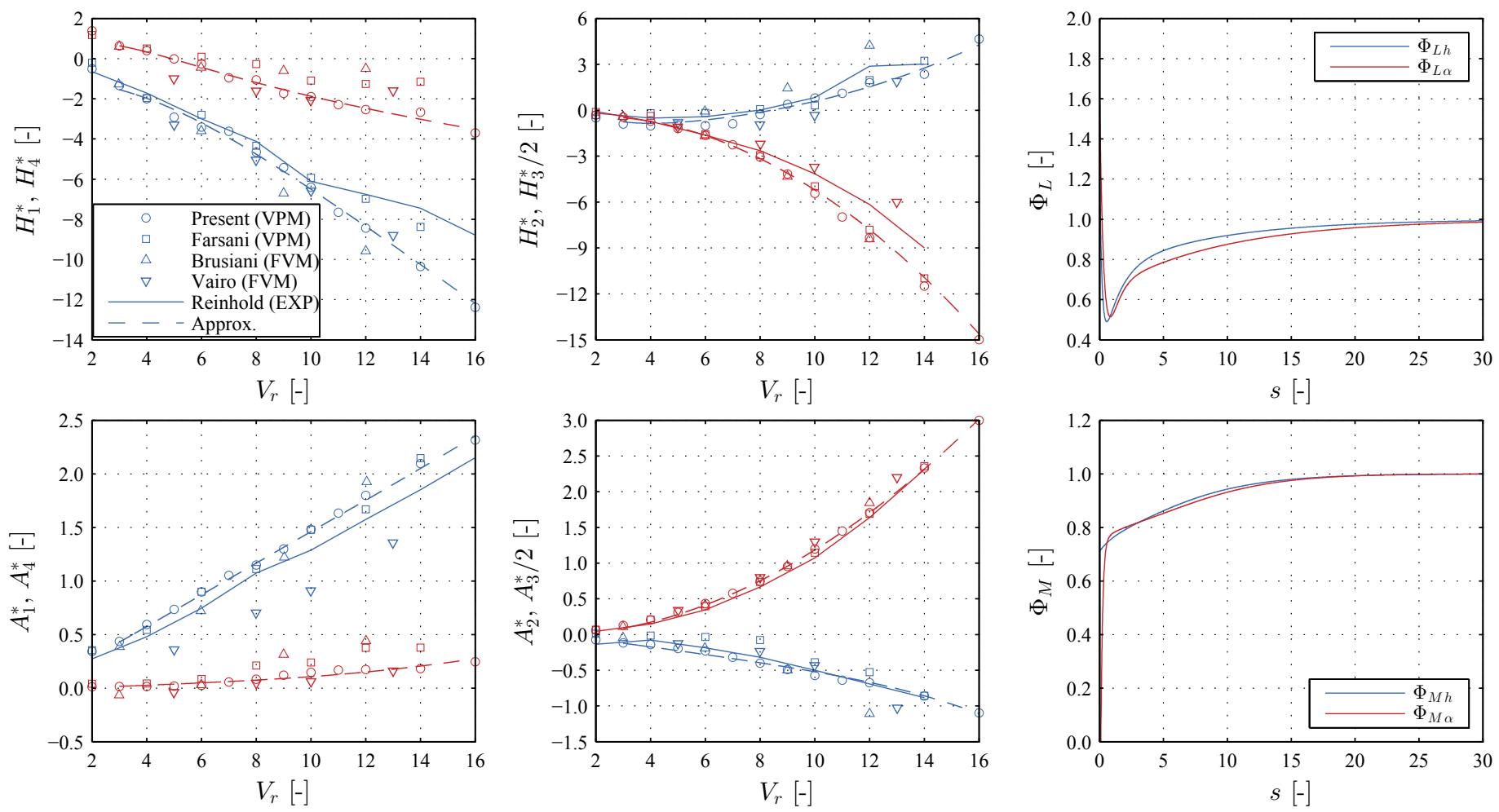

Figure 9: Flutter derivatives compared with former results (Brusiani et al., 2013; Farsani et al., 2014; Reinhold et al., 1992; Vairo, 2003) and their rational approximation (Approx.) for $\alpha_{s}=0 \mathrm{deg}$ (left and center): $H_{1}^{*}, H_{2}^{*}, A_{1}^{*}, A_{2}^{*}$ (blue) and $H_{4}^{*}, H_{3}^{*}, A_{4} *, A_{3}^{*}$ (red). The corresponding indicial functions: lift $\Phi_{L}$ (top-right) and moment $\Phi_{M}$ (bottom-right). The abbreviations in the legend are given in the caption of Tab. 2.
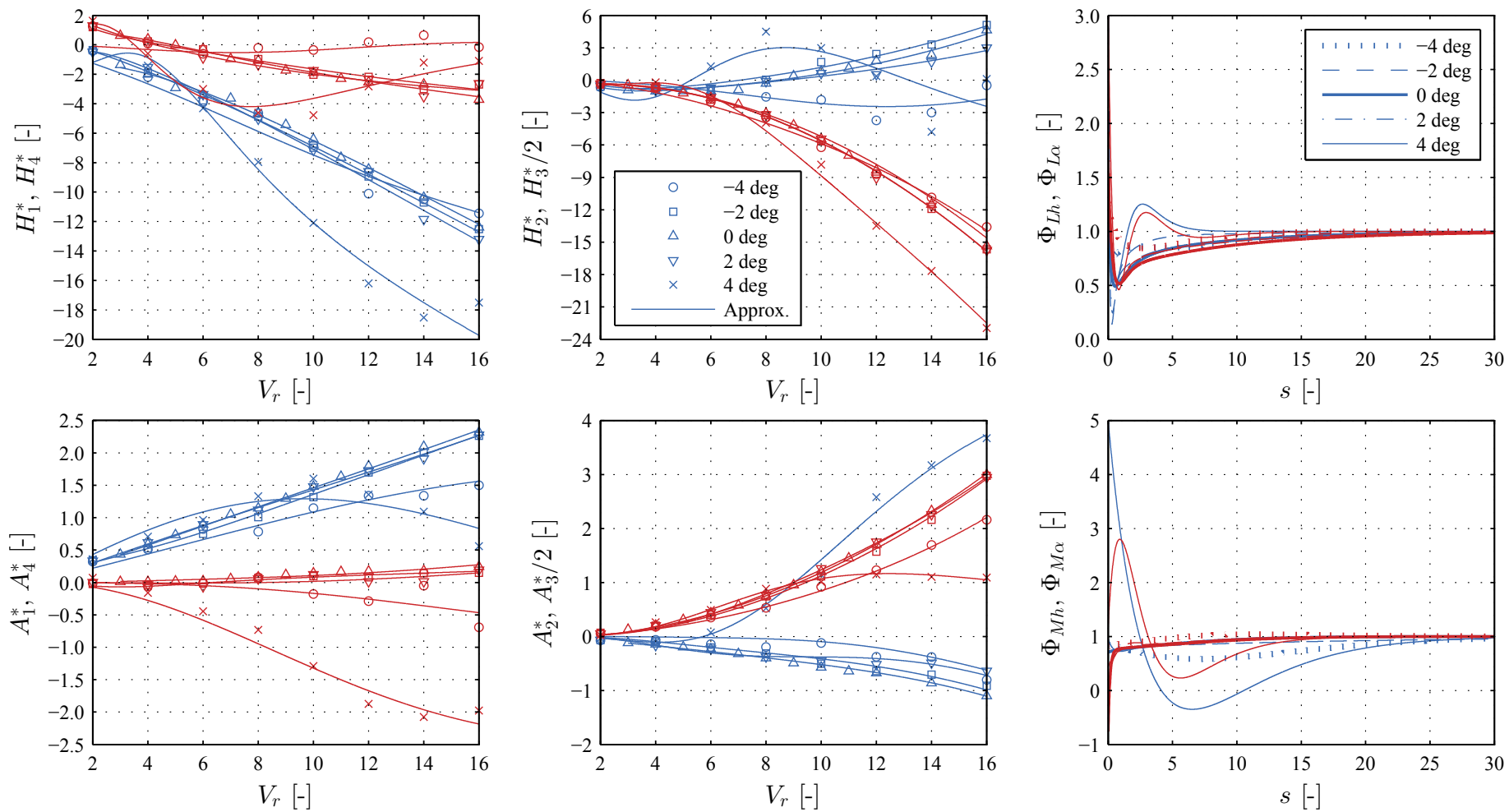

Figure 10: Flutter derivatives for varying angle of attack and their rational approximation (Approx.) (left and center): $H_{1}^{*}$, $H_{2}^{*}, A_{1}^{*}, A_{2}^{*}$ (blue) and $H_{4}^{*}, H_{3}^{*}, A_{4}^{*}, A_{3}^{*}$ (red). The corresponding indicial functions: lift (top-right) for $\Phi_{L h}$ (blue) and $\Phi_{L \alpha}$ (red) and moment (bottom-right) for $\Phi_{M h}$ (blue) and $\Phi_{M \alpha}$ (red).

erroneous high amplitudes in the displacements for the HNL model. Therefore, the flutter derivatives in the $\alpha_{s}= \pm 4$ deg range are used for the HNL model. 

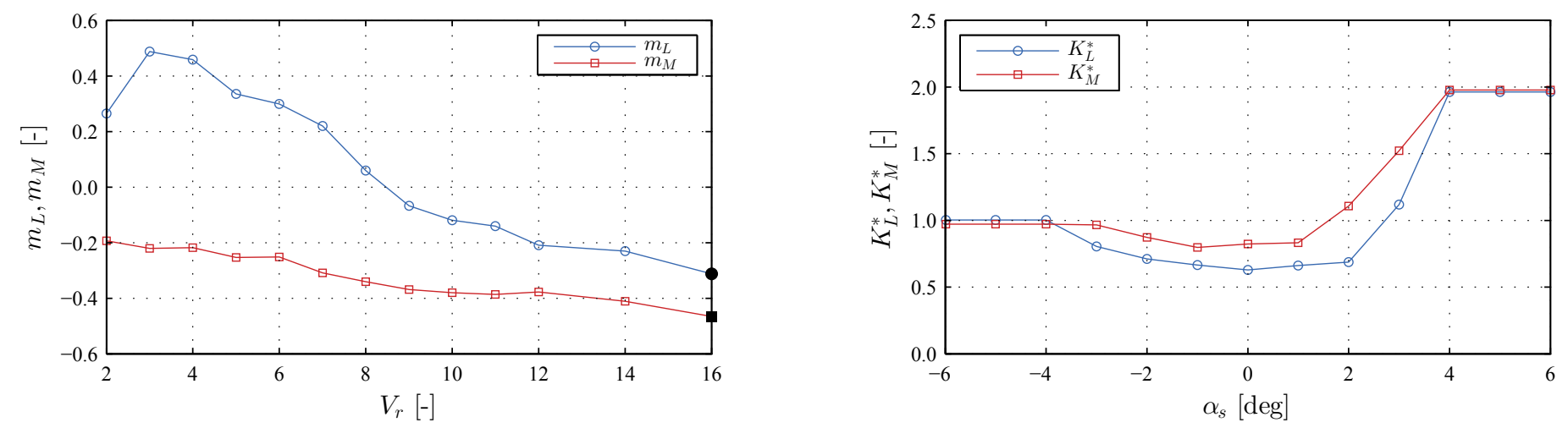

Figure 11: Aerodynamic center $m_{L}$ and $m_{M}$ for the lift and moment forces, respectively (left). The selected values for the analyses are indicated by the black-filled markers. Frequency-dependent correction coefficients $K^{*}$ for $V_{r}=12$ (right).

The aerodynamic center is an important parameter in the quasi-steady based models. Figure 11 depicts the aerodynamic center and the correction coefficients for the selected case, based on the flutter derivatives (see Eqs. (13)). It can be observed from the figure that the moment aerodynamic center $m_{M}$ is in the range between -0.2 and -0.45 with a convergent trend beyond -0.4. The lift aerodynamic center $m_{L}$ changes from positive to negative due to the changing value of the $H_{2}^{*}$ derivative (see Fig. 9). Commonly for bridge decks, reduced velocities beyond $V_{r} \geq 15$ are considered to represent an equivalent quasi-steady state; hence, the aerodynamic center is selected in this reduced velocity range (Diana et al., 2013). In this case, the values for the aerodynamic center in the subsequent analyses are selected for $V_{r}=16$ and they fall the reasonable range between -0.5 and 0.5 given by $\mathrm{Wu}$ and Kareem (2013). The selected values for the aerodynamic center can strongly influence the results in the aeroelastic analysis, by increasing or decreasing the contribution of aerodynamic damping due to rotation. Choosing a large positive value for the moment aerodynamic center may lead to premature torsional flutter for streamlined bridge decks (Wu and Kareem, 2013). For bluff bridge decks prone to torsional flutter, obtaining the aerodynamic center at very high reduced velocities may lead to failure of capturing a torsional flutter (Kavrakov and Morgenthal, 2017). Although obtaining the aerodynamic center from the flutter derivatives may not be accurate for a nonlinear model (Wu et al., 2013), presently the authors are not aware of other standard procedure for its determination. Herein, parametric studies on the influence of the aerodynamic center on the response are beyond the scope of the study and further discussions are available in Diana et al. (2013); Kavrakov and Morgenthal (2017); Wu and Kareem (2013); Wu et al. (2013). The frequency-dependent correction coefficients $K_{L}^{*}$ and $K_{M}^{*}$ in the CQS model (see Fig. 11, right) can reduce or increase the slope of the static wind coefficients, based on the aerodynamic characteristics described by the flutter derivatives. In this case, these coefficients attain high value for positive angles of attack due to the prominent nonlinearity, as noted previously. Beyond the range of $\pm 4 \mathrm{deg}$, these coefficients are maintained as constant.

The aerodynamic admittance is computed by rearranging the spectral form of Eqs. (7) and using the PSDs of the buffeting forces and wind velocity fluctuations for time amounting to $s=400$ chords. In Fig. 12, the computed aerodynamic admittance functions are shown along with the complex approximation of the Sears admittance function (Scanlan, 2000) for $\alpha_{s}=-2,0$ and $2 \mathrm{deg}$. The PSDs of the wind velocity fluctuations are determined at $(0,0)$ in the domain from a simulation considering only the free-stream turbulence, that is, without a section in the domain. As the same seed is selected in the stochastic random walk procedure (see Sec. 3.1), the wind fluctuations are deemed reproducible between simulations. If the wind spectrum is determined in such manner, the influence of the section on the incoming flow is not taken into account. However, the LU and HNL model cannot capture this effect anyway. As a separation between the influence of the longitudinal and vertical fluctuations in the buffeting forces is not feasible with the present method for determination of the aerodynamic admittance, it is assumed that $\chi_{L}=\chi_{L u}=\chi_{L w}$ and $\chi_{M}=\chi_{M u}=\chi_{M w}$.

Two regions are distinguished in the aerodynamic admittance with respect to the reduced velocity: below and above the cutoff reduced velocity (see Fig. 12). Above the cutoff reduced velocity the admittance is governed by the incident wind fluctuations, while the wake fluctuations govern the admittance below the cutoff reduced velocity. The latter observation can be linked to the Strouhal number as well, as the vortex shedding frequency corresponds to the peak of the admittance below the cutoff reduced velocity. There are various interpretations of the aerodynamic admittance for a bridge deck. Kareem and $\mathrm{Wu}$ (2013) separate three distinctive regions of the admittance, characterized by the incident, shear layer or near wake and the wake fluctuations. The LU and HNL models, which model for the fluid memory in the buffeting forces, account only for the region of the aerodynamic admittance governed by the incident fluctuations. Therefore, only the part of the aerodynamic admittance above the cutoff reduced velocity is used for the rational approximation. There is no clear indication for the selection of the cutoff reduced velocity; rather a heuristic distinction where the three regions are divided. In this case for the cutoff limit is chosen at $V_{r}=2$. It should be noted that the incident fluctuations also have contribution to the admittance below the cutoff reduced velocity. However, this contribution is small compared to the one from the wake fluctuations. Since with this method the phase and magnitude component of the aerodynamic admittance cannot be obtained separately, it is assumed that the imaginary part $G^{*}$ from the complex form of the admittance is the same as 

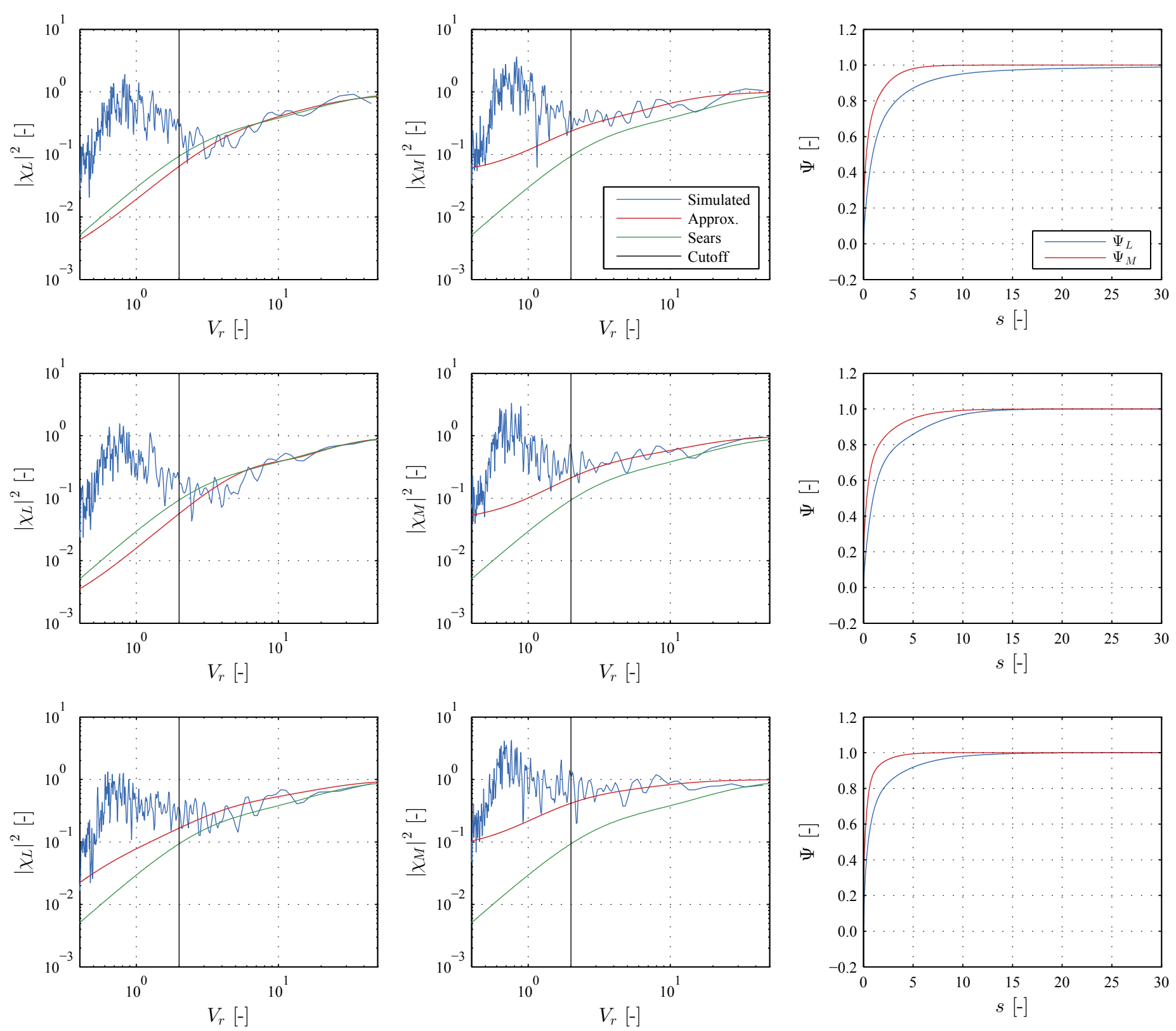

Figure 12: Aerodynamic admittance $\chi$ and its rational approximation (Approx.) for lift (left) and moment (center). The cutoff frequency describes the lower limit for the approximation in order to eliminate the vortex shedding effects. The corresponding indicial functions $\Psi$ are shown (right). The admittance is determined for varying angle of attack: $\alpha_{s}=-2$ $\operatorname{deg}$ (top), $\alpha_{s}=0 \operatorname{deg}$ (center) and $\alpha_{s}=2 \operatorname{deg}$ (bottom).

the one in the complex Sears approximation (Scanlan, 2000). The indicial functions of the buffeting forces are relatively smooth with short rise-time and are depicted in Fig. 12. The shear layer and wake fluctuations significantly influenced the aerodynamic admittance for angles higher than $2 \mathrm{deg}$ and lower than $-2 \mathrm{deg}$; therefore, the region of the admittance governed by incident fluctuations could not be clearly distinguished for angles outside this range. In case of the HNL model, the linearization of the buffeting forces is bounded in the $\alpha_{s}= \pm 2$ deg interval.

\subsection{Buffeting Forces}

In this section, the fluctuating wind coefficients (time-dependent moment and lift coefficients) are studied under turbulent free-stream for a static section in order to single out the discrepancies between the CFD, chosen to be the reference model, and semi-analytical models for the buffeting forces. As the Reynolds number is kept constant, both cases of low $(I=6 \%)$ and high $(I=10 \%)$ turbulence intensity are studied at constant mean wind speed of $U=30 \mathrm{~m} / \mathrm{s}$. The input wind fluctuations for the semi-analytical models are the simulated wind time-histories at $(0,0)$ in the domain without a section.

In Fig. 13, a representative part of the time-histories of the fluctuating wind coefficients is presented for the CFD and semi-analytical models. It is observed that the high-frequency component of the fluctuating wind coefficients is overestimated by the LQS model, as the peaks in the time-histories are significantly higher than the ones for the reference model. 

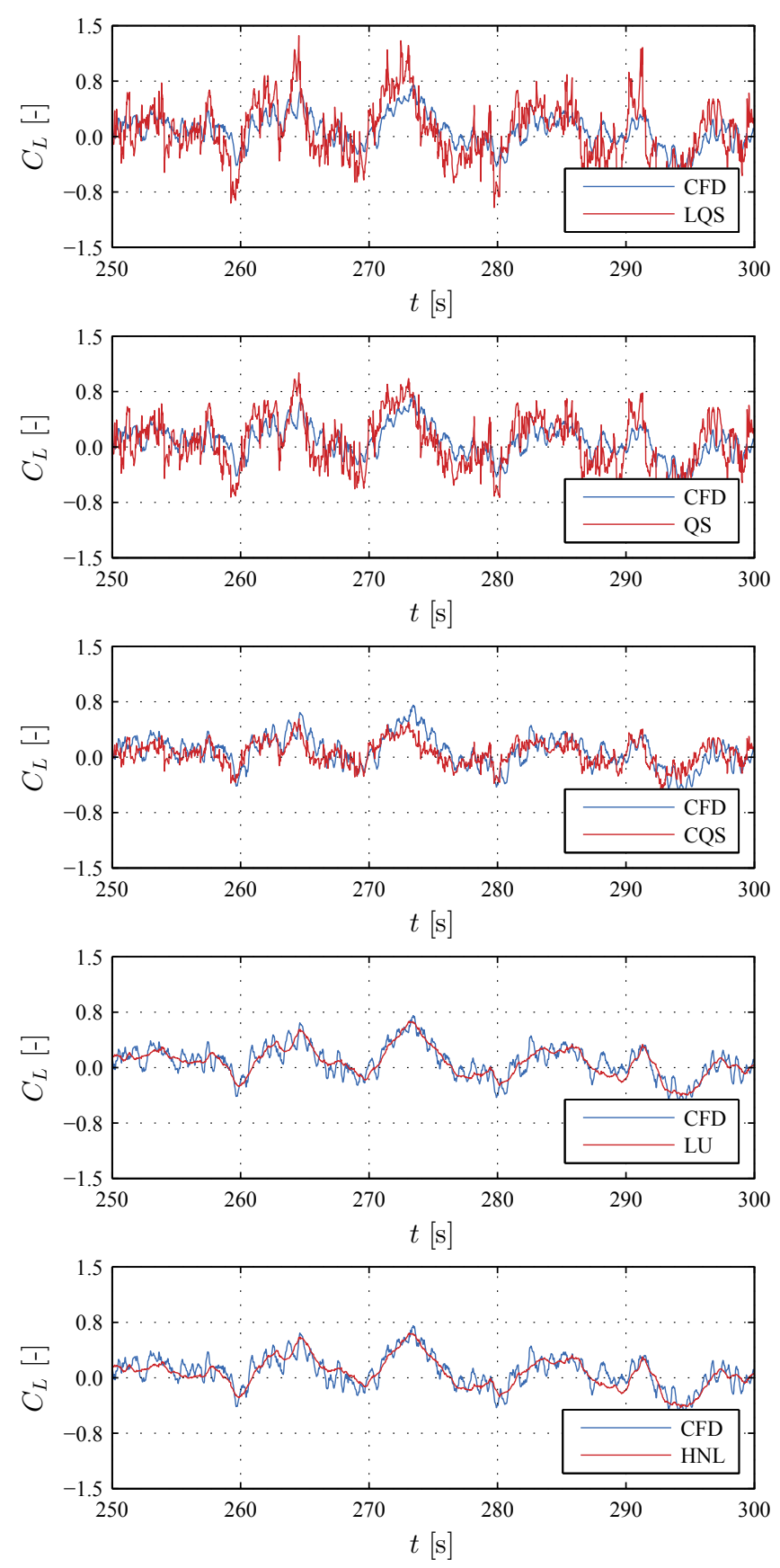
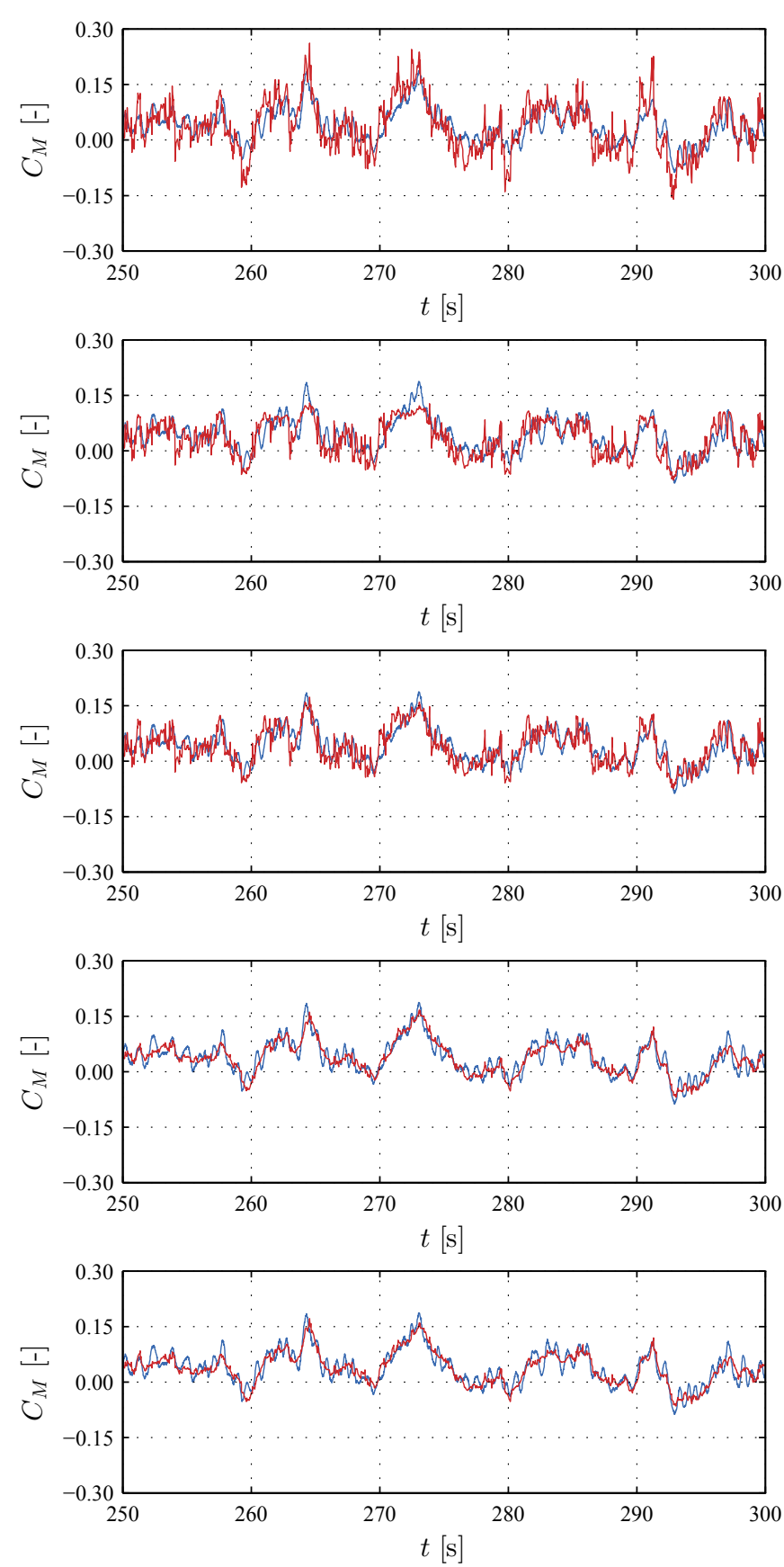

Figure 13: Representative sample time-histories of the lift $C_{L}$ (left) and moment $C_{M}$ (right) coefficients for the CFD and semi-analytical models. The simulated wind fluctuations at $(0,0)$ are used for the semi-analytical models at $U=30 \mathrm{~m} / \mathrm{s}$ and $I=6 \%$.

Nonetheless, the low-frequency content of the fluctuating wind coefficients for the LQS model follows the same trend like the CFD model. The peaks in the fluctuating wind coefficients for the QS model have lower amplitudes compared to the LQS model, which effectively shows the influence of the quasi-steady aerodynamic nonlinearity on the buffeting forces for large effective angles of attack. Nevertheless, the effect of nonlinearity on the buffeting forces is overestimated by the QS model, which is observed when the time-histories for this model are further compared to the ones for the CFD model (see e.g. $C_{M}$ for $t=270-280 \mathrm{~s}$ ). Although the additional high-frequency content in the fluctuating wind coefficients is still overestimated for the CQS model, a better correspondence is achieved between this and the reference model compared to the QS and LQS models. The averaged fluid memory considered in the CQS model is dependent on the correction coefficient $K^{*}$ (Eqs. (10)), which is based on the flutter derivatives. When there is no motion, the fluid memory of the buffeting forces is described by the aerodynamic admittance; therefore, the correction coefficient should be based on the admittance. However, the buffeting and self-excited part of the effective angle of attack are inseparable in a nonlinear formulation and thus, the correction coefficient $K^{*}$ is computed from the flutter derivatives. Mainly, the LU and HNL models are in the best agreement with CFD model for the fluctuating wind coefficients. The effect of fluid memory is 

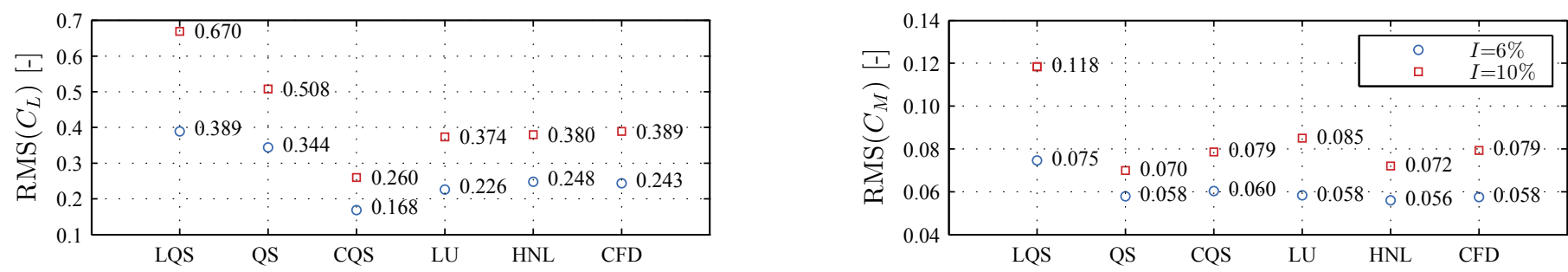

Figure 14: RMS of the lift $C_{L}$ (left) and moment $C_{M}$ (right) coefficients for the CFD and semi-analytical models at $U=30$ $\mathrm{m} / \mathrm{s}$ and $I=6 \%$.

a)

c)
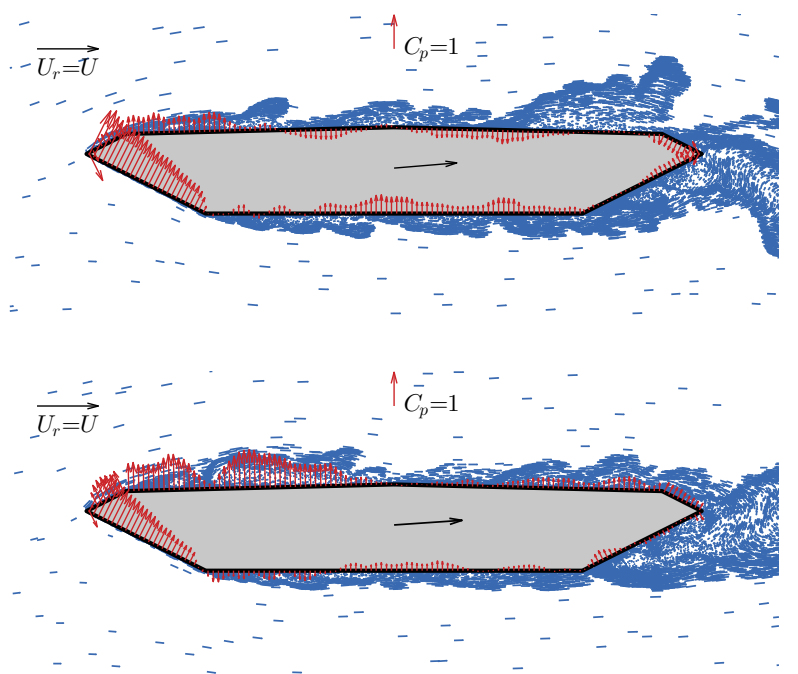

b)

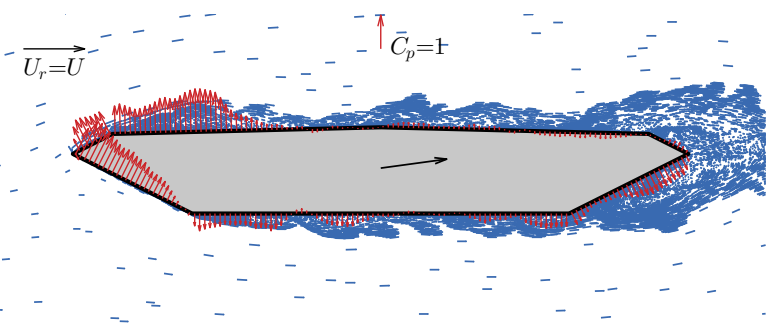

d)

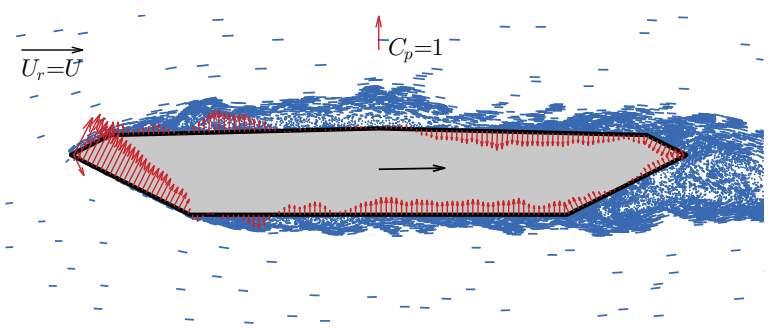

Figure 15: Vortex particle map, pressure $C_{p}$ (red arrows) distribution due to incoming vertical gust at $U=30 \mathrm{~m} / \mathrm{s}$ and $I=6 \%$. The snapshots correspond to: a) $t=259.40 \mathrm{~s}$; b) $t=264.04 \mathrm{~s}$; c) $t=264.31 \mathrm{~s}$ and d) $t=265.06 \mathrm{~s}$ from Fig. 13 . The black arrow corresponds to both direction and magnitude of the resultant wind velocity $U_{r}$ (see Eqs. $(4,5)$ ).

manifested as a filter for the fluctuating wind coefficients, resulting in their amplitude and phase modulation. Looking at the time-histories for these models, it is evident that an additional high-frequency component is present in the fluctuating wind coefficients for the CFD model, which is revisited later in this section.

Generally, the observations made from the qualitative assessment of the time-histories correspond to the quantitative assessment of the root mean square (RMS) of the fluctuating wind coefficients for both turbulent cases (see Fig. 14). The RMS of the fluctuating wind coefficients for the LU and HNL model correspond well with the RMS for the CFD model. By including the quasi-steady nonlinearity of the low-frequency wind fluctuations in the HNL model, the RMS of the lift coefficient is slightly increased, while a decrease is noted in the RMS of the moment coefficient. Comparing the results for the LQS and LU model, it can be seen that fluid memory plays a major role in the buffeting forces, particularly in the high turbulence case. The impact of the quasi-steady nonlinearity is prominent for the moment coefficient, which is observed from the results of the QS model in contrast to the LQS model. As discussed previously, the effect of the nonlinearity may be overestimated if the fluid memory is not taken into account concurrently for the buffeting forces. Therefore, the good correspondence in the RMS of the moment coefficient for the QS and CQS models with the CFD model could be arbitrary.

In order to further examine the discrepancies between the fluctuating wind coefficients for the LU and CFD model, a sequence of snapshots of an instantaneous particle map and corresponding pressure distribution is depicted in Fig. 15. The sequence of snapshots corresponds to the peak moment coefficient for $t=264-265 \mathrm{~s}$. An incoming vertical gust (given by the resultant velocity vector $U_{r}$ ) initiates a flow separation on the top surface (see Fig. 15, a), which grows in size as a vortex is entrained. The gust velocity reaches a peak almost simultaneously with the suction pressure at the top surface near the leading edge (see Fig. 15, b). The vortex is then convected downstream and a secondary vortex is formed, resulting in an additional suction pressure (see Fig. 15, c). Both of the vortices are then convected downstream (see Fig. 15, d). Since all of the semi-analytical models resulted in a peak in the fluctuating moment coefficient (see Fig. 13), the main influence in the moment force is due to the incident fluctuations. Nevertheless, the peak of $C_{M}$ for the LU model is slightly reduced compared to the one for the CFD model, which may originate from some local effects. Fundamental studies on long rectangular cylinders note that the unsteady behavior of separation bubble results in quasiperiodic fluctuating pressures as the vortex is convected downstream (Cherry et al., 1984). For a turbulent free-stream, the magnitude of these fluctuating pressures increases significantly (Saathof and Melbourne, 1997) and the length of the separation bubble reduces (Hiller and Cherry, 1981). The local effects are highly influenced by the three-dimensionality 

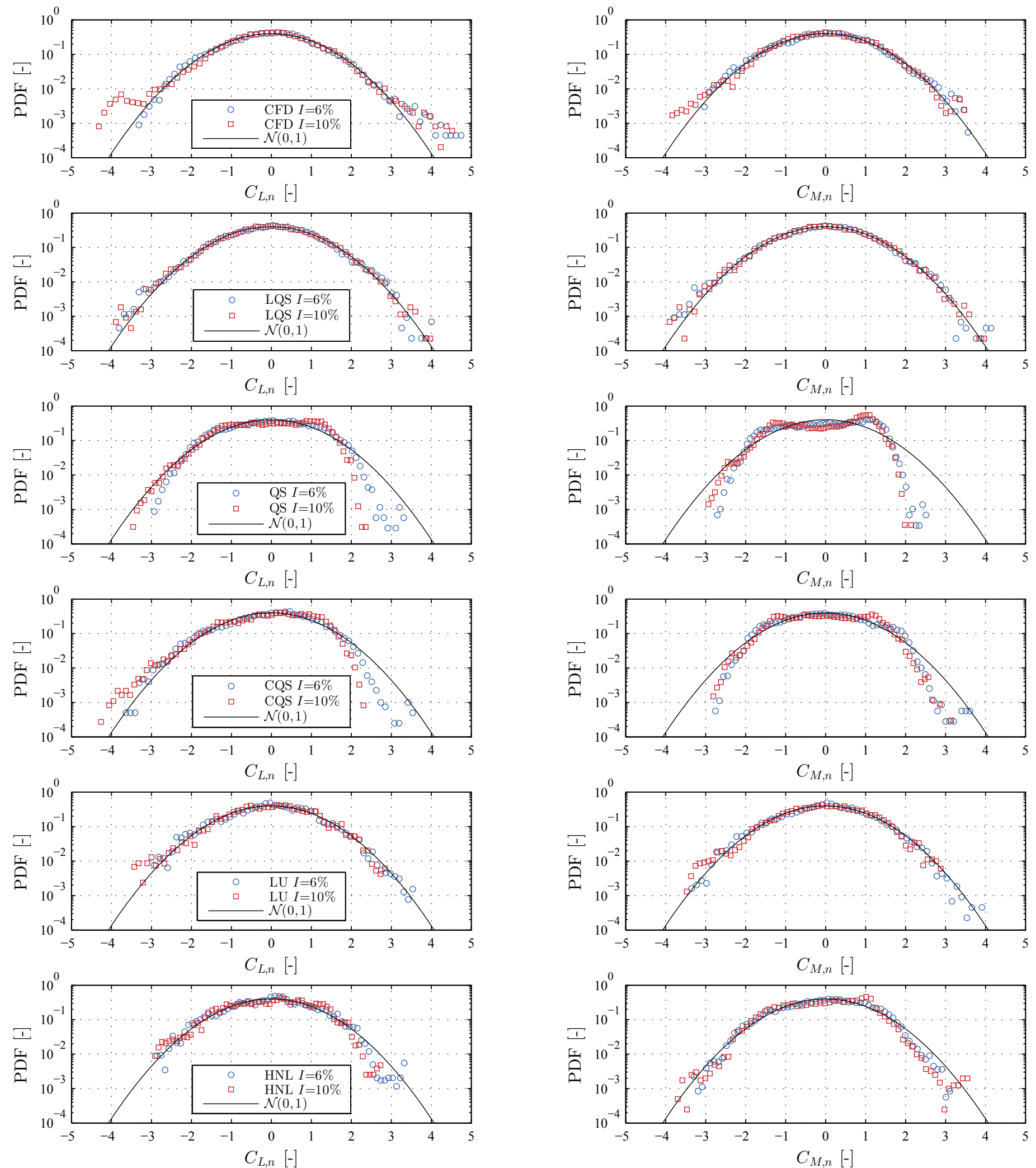

Figure 16: PDF of the normalized (standard score) lift $C_{L, n}$ (left) and moment $C_{M, n}$ (right) coefficients at $U=30 \mathrm{~m} / \mathrm{s}$ and $I=6 \%$. 

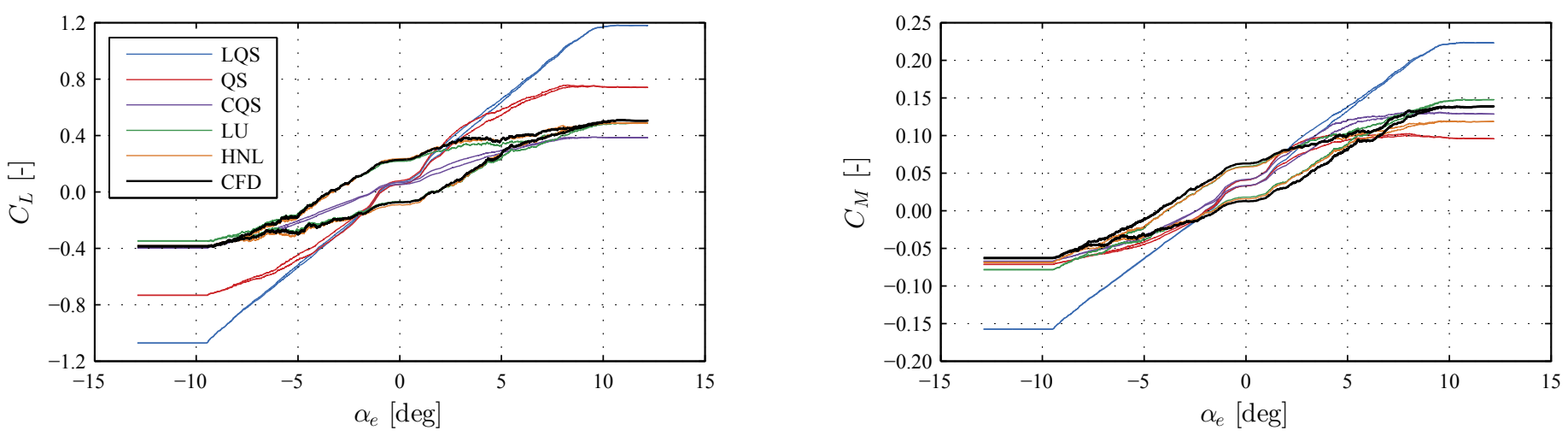

Figure 17: Filtered envelope of the aerodynamic hysteresis of the lift $C_{L}$ (left) and moment $C_{M}$ (right) coefficients at $U=30 \mathrm{~m} / \mathrm{s}$ and $I=6 \%$.

of the flow, and therefore they are not well captured by the current CFD model. Presently, it is not well understood how the local 2D effects influence the local pressure. Furthermore, these local effects are length scale dependent. The experimental studies are conducted for moderate Reynolds number with significantly lower length scale to depth ratio $L_{u} / H \approx 2.1$ compared to this study $L_{u} / H \approx 25$. Therefore, strong conclusions on the local effects cannot be drawn without further fundamental investigations.

It is noted that the combined effects due to the nonlinearity and non-stationarity are intractable by the selected semianalytical models. Although the underlying mechanisms of bluff-body aerodynamics are still not fully established, three effects could be mentioned, which are the probable cause for the high frequency content in the fluctuating wind coefficients for the CFD model, namely: (i) unsteady aerodynamic nonlinearity, (ii) local effects due quasi-periodic fluctuations and (iii) vortex shedding. As the trend of the fluctuating wind coefficients for the LU and HNL model is similar and the highfrequency fluctuations of CFD model are mostly quasi-periodic, the claim that these fluctuations are due to the vortex shedding and local turbulence effects seems plausible. Nevertheless, it is difficult to single out these effects. The influence of the vortex shedding can be also seen in the aerodynamic admittance (see Fig. 12).

To further study the effect of aerodynamic nonlinearity, the PDFs of the fluctuating wind coefficients are shown in Fig. 16. For wind fluctuations that follow the Gaussian PDF (see Fig. 6), a linear force model would also result in a similar PDF of the fluctuating wind coefficients, which is the case for the LU model. The PDFs of the fluctuating wind coefficients for the CFD model generally follow the trend of the Gaussian PDF for the low turbulence case, except for the positive values of the lift coefficient at the tail of the distribution. While the values at the tail for the CFD model can be due to the vortex shedding and/or aerodynamic nonlinearity, it can be seen that the PDF of the lift and especially the moment coefficient for the QS and CQS models significantly depart from the Gaussian trend. This departure fortifies the claim that the effect of nonlinearity on the buffeting forces is overestimated for the QS and CQS model.

Besides modulation of the amplitude of the buffeting forces, the consideration of the fluid memory also affects the phase between the buffeting forces and wind fluctuations. Figure 17 depicts the envelope of the fluctuating wind coefficients with respect to the instantaneous angle of attack for the low turbulence case, filtered using a moving average filter with 30 samples. It is evident that the hysteretic behavior of the fluctuating wind coefficients, appearing for the CFD model, can be only captured by the LU and HNL models. The good correspondence of these models indicates that using the imaginary part for the aerodynamic admittance from the Sears' function is justified. However, this assumption is case-dependent. The consideration of the phase in the complex form of the aerodynamic admittance can significantly influence the response in aeroelastic analyses at high reduced velocities when the intermodal coupling is meaningful, as noted by Chen (2006).

\subsection{Aerostatic Analysis}

The aerostatic response is computed for a smooth free-stream at the selected wind speeds using CFD, semi-analytical linear and nonlinear aerostatic analyses. The latter analysis is performed by iterative updating of the static wind coefficients with respect to the position of the section until an aerostatic equilibrium is achieved. For the CFD analysis, the section is subjected to a smooth free-stream and undergoes free oscillations with a duration of $s=160$. The displacements are then averaged for $s=110-160$, for which the initial impulse is sufficiently damped out. In Fig. 18, the displacements of the vertical and torsional degrees of freedom are given. The figure suggests that nonlinear aerostatic analysis is required for high wind speeds. Similar observations are noted by Zhang et al. (2002), where the influence of the geometric and aerodynamic nonlinearities on the flutter velocity are studied. The latter will be discussed in Sec. 4.6. It is noted that for the linear models, the linearization of the static wind coefficients is performed at the angle of nonlinear static equilibrium. 

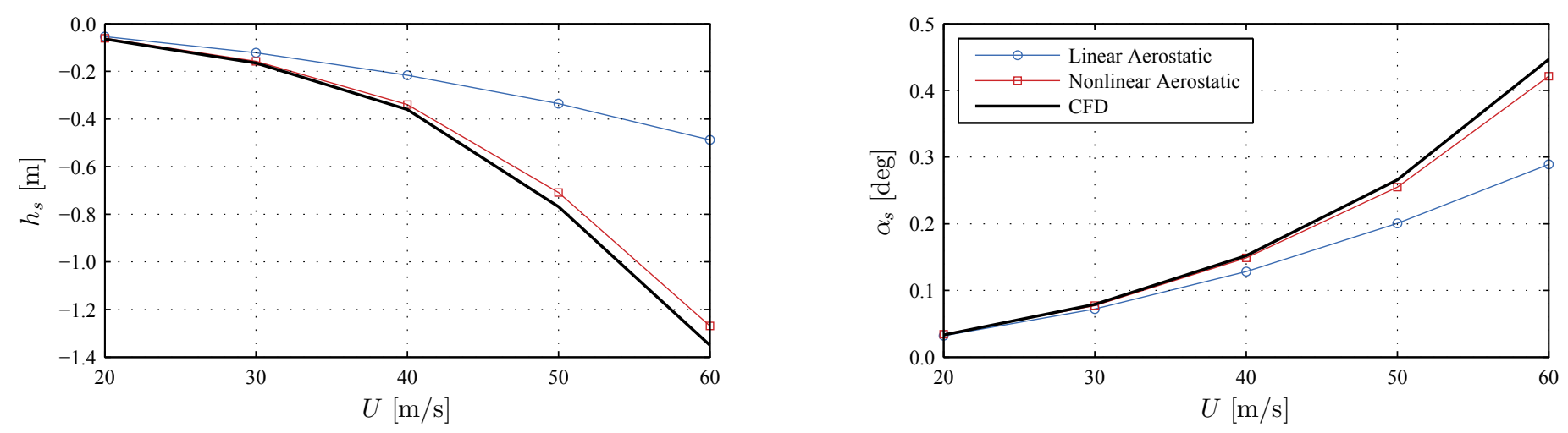

Figure 18: Comparison between semi-analytical and CFD aerostatic response under smooth free-stream for the vertical displacements $h_{s}$ (left) and rotation $\alpha_{s}$ (right).

\subsection{Aeroelastic Analysis}

Aeroelastic analyses are performed for velocities ranging from 20 up to $60 \mathrm{~m} / \mathrm{s}$ for both turbulent cases. Since the turbulent Reynolds number is constant, the influences of the reduced velocity, turbulence intensity and motion amplitudes on the dynamic response are studied. Figure 19 depicts snapshots of the velocity field during oscillations of the deck. It is evident that the velocity of the free-stream turbulence is lower than the velocity in the wake. RMS values of the vertical and torsional displacements are given in Fig. 20 for the selected wind speed range for both turbulent cases. Specific values of the response are listed in Fig. 21 for two representative velocities: high $(U=30 \mathrm{~m} / \mathrm{s})$ and low $(U=60 \mathrm{~m} / \mathrm{s})$ with respect to the flutter limit $U_{c r} \approx 70 \mathrm{~m} / \mathrm{s}$. Again, the CFD model is taken as a reference model.

Based on the figures containing the RMS of the displacements, it is observed that the aeroelastic response for the LQS model is overestimated compared to the one for the CFD model for all of the cases. If the response for the LQS model is further compared to the results of the LU and QS models, it is evident that the effects of linear fluid memory and quasi-steady nonlinearity decrease the displacement amplitudes, particularly for the case with high turbulence intensity. The discrepancy between the vertical responses for the QS and CFD models is decreasing for higher wind velocities. While the good correspondence between these two models can be attributed to the fact that the effect of fluid memory becomes negligible at high reduced velocity, this might not be the case herein. The effect of the nonlinearity, that is considered in the QS model, on the aeroelastic response is likely overestimated, as discussed for the buffeting forces in Sec 4.3. Therefore, the good correspondence of the vertical displacements for these models could be arbitrary. Another indication for the incapability of the QS model to represent the nonlinear aeroelastic behavior is the significant difference in the rotation with respect to the CFD model for high wind speeds, particularly for the case with high turbulence. The trend of the response for the CQS model is essentially the same as the one for the QS model compared to the CFD model. For the case with low turbulence intensity, the vertical displacements for the CQS model are in better agreement with the response for the CFD model than the QS model. Other than this case, there is no clear indication how the averaged fluid memory affects the aeroelastic response. Although no conclusive statements can be made on this account, the abrupt changes in the correction coefficient in the CQS model might result in an irregularity in the trend of the displacements, as noted by Wu and Kareem (2013). The same observations regarding effect of quasi-steady nonlinearity in the QS model hold for the CQS model as well. Even though the aim of the MQS model is to better describe the aerodynamic damping than the LQS model, an increased discrepancy for the vertical displacements is noted for the MQS model compared to the LQS model. This is a result of the quadratic trend of $H_{2}^{*}$, in contrast to the linear trend assumed in the MQS model (Øiseth et al., 2010). The slope of the RMS of the torsional response with respect to the mean wind speed for the MQS model is lower than the one for the CFD model, which indicates insufficient quality of approximation for the torsional damping. This can be also observed in Fig. 21 as the rotational displacements for the MQS model are overestimated at $U=30 \mathrm{~m} / \mathrm{s}$
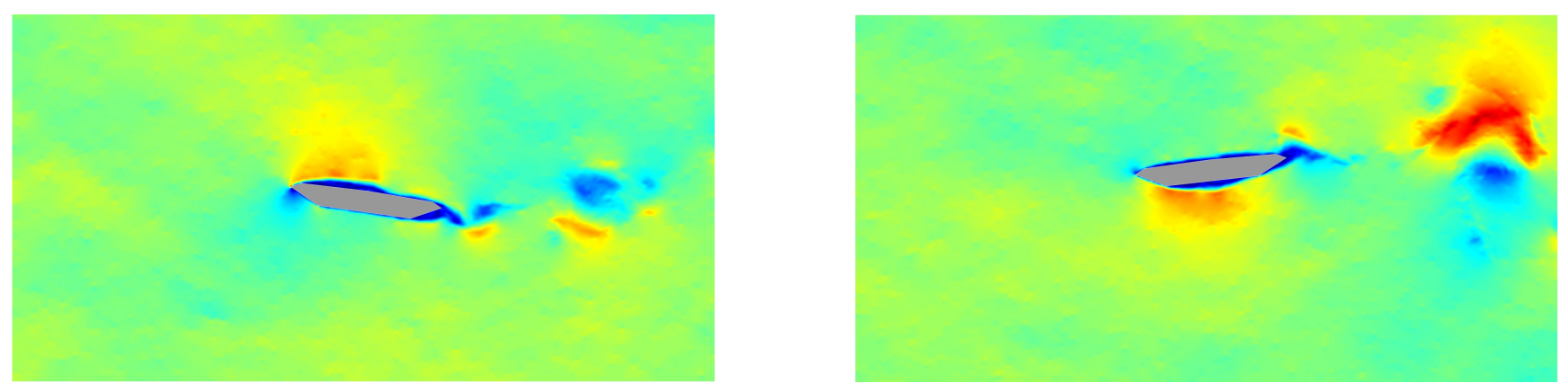

Figure 19: Instantaneous velocity fields of the aeroelastic analysis at $U=30 \mathrm{~m} / \mathrm{s}$ and $I=6 \%$. 

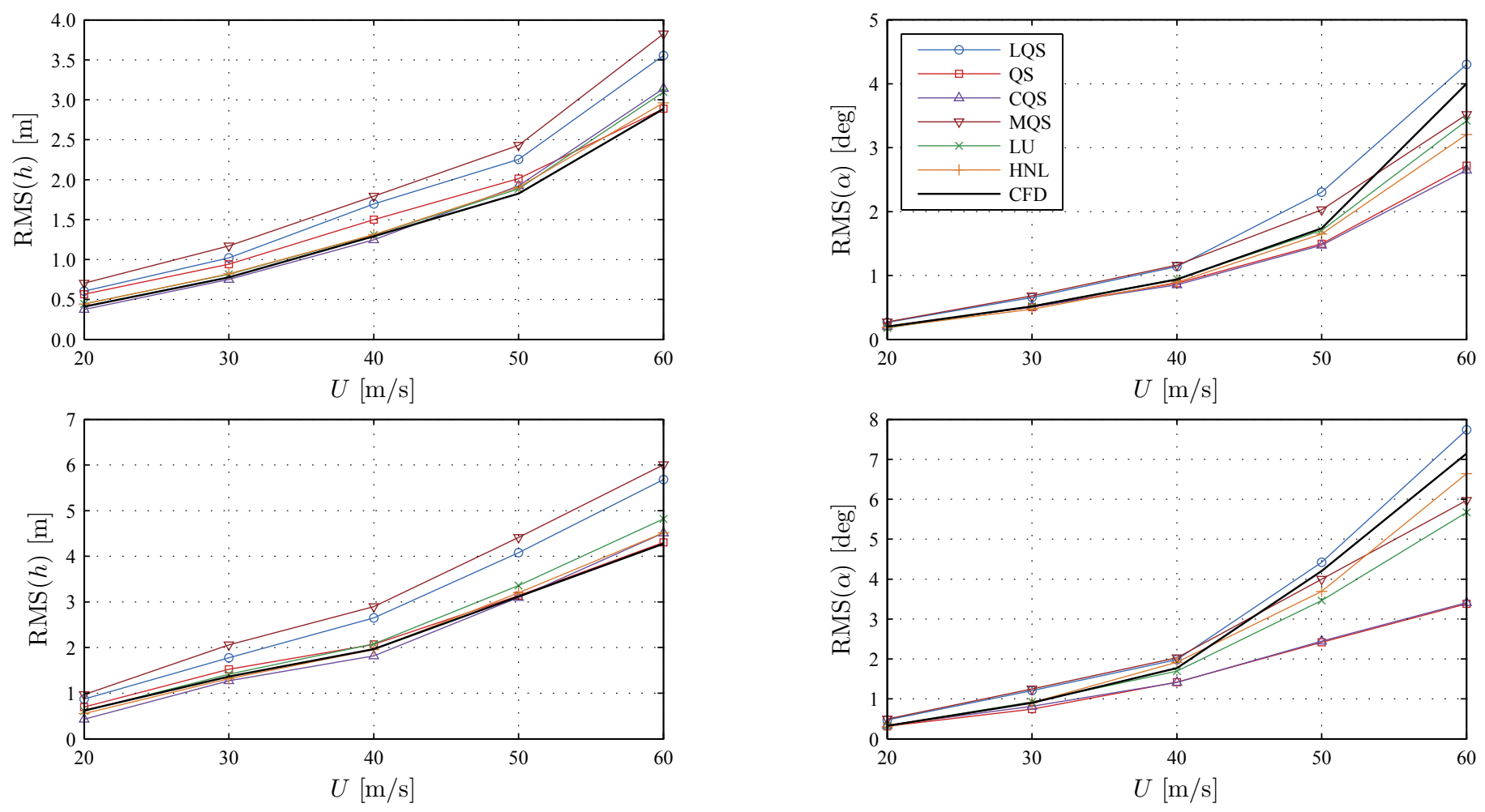

Figure 20: RMS of the vertical displacements $h$ (left) and rotation $\alpha$ (right) for the CFD and semi-analytical models for $I=6 \%$ (top) and $I=10 \%$ (bottom).
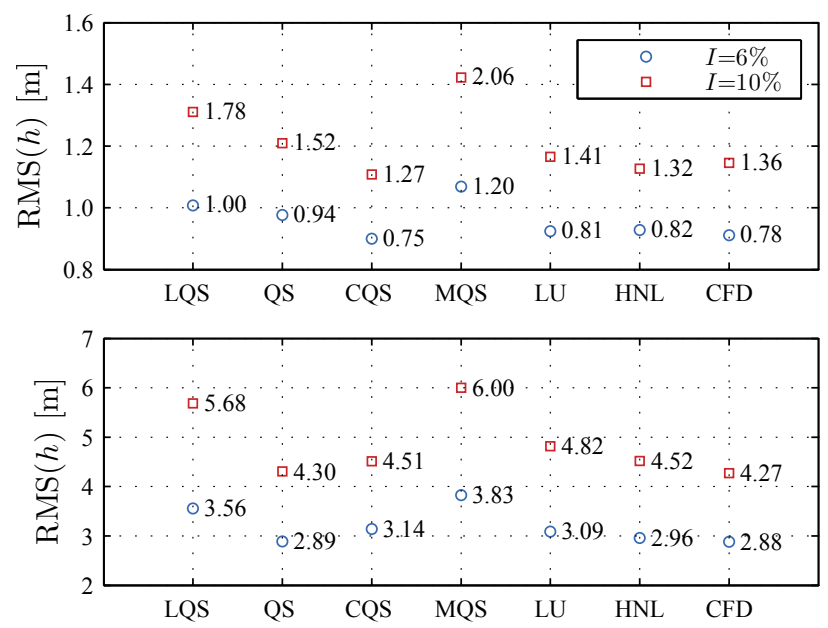
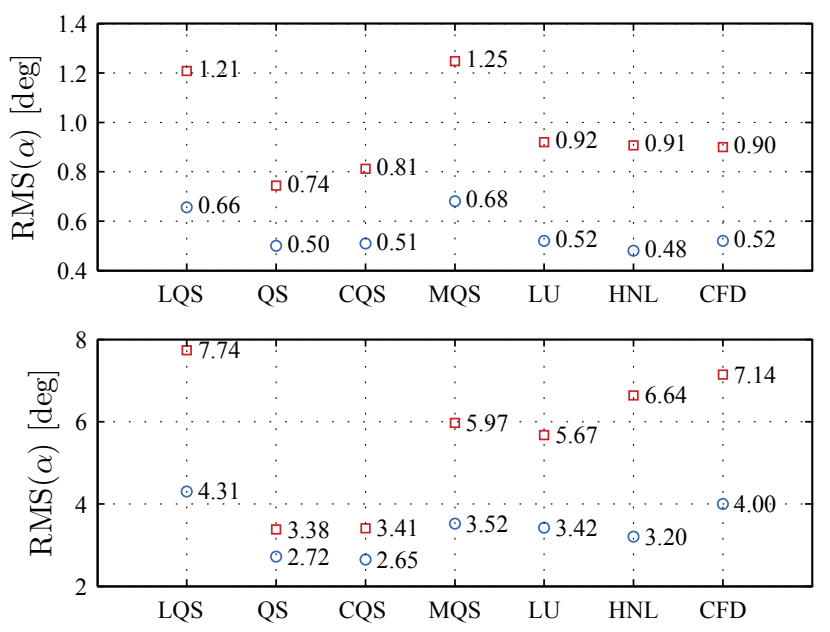

Figure 21: RMS of the vertical displacements $h$ (left) and rotation $\alpha$ (right) for the CFD and semi-analytical models at $U=30 \mathrm{~m} / \mathrm{s}$ (top) and $U=60 \mathrm{~m} / \mathrm{s}$ (bottom).

and underestimated at $U=60 \mathrm{~m} / \mathrm{s}$ with respect to the CFD model. Øiseth et al. (2010) used the secant approximation of the torsional flutter derivatives to address this issue; however, this is not considered in this study.

Generally, the smallest discrepancies in the response are obtained for the LU and HNL models with respect to the CFD model, taking into account all considered cases and both degrees of freedom. Representative time-histories of the aeroelastic response for the semi-analytical models are compared with the response for the CFD model in Fig. 22, for the case with low turbulence and $U=30 \mathrm{~m} / \mathrm{s}$. The figure suggests that the response for the CFD model lags behind all models based on the quasi-steady assumption. This indicates the phase effect of the fluid memory in the aerodynamic forces. Based on a qualitative assessment, the response time-histories for the LU and HNL models appear to be in the best agreement with the response time-histories for the CFD model.

A particular feature is observed in the torsional response for the CFD model. Above a certain velocity $(U=50 \mathrm{~m} / \mathrm{s}$ and $U=40 \mathrm{~m} / \mathrm{s}$ for the low and high turbulence case, respectively), a distinctive change of the slope of RMS of the response is noted (see Fig. 21). For both levels of turbulence, a notable similarity in the torsional responses is the amplitude at which this change of slope occurs $(\operatorname{RMS}(\alpha) \gtrsim 3 \mathrm{deg})$. When a motion is introduced to an aeroelastic system in addition to the free-stream turbulence, it is even more challenging to describe the origin of discrepancies in the aerodynamic forces for 

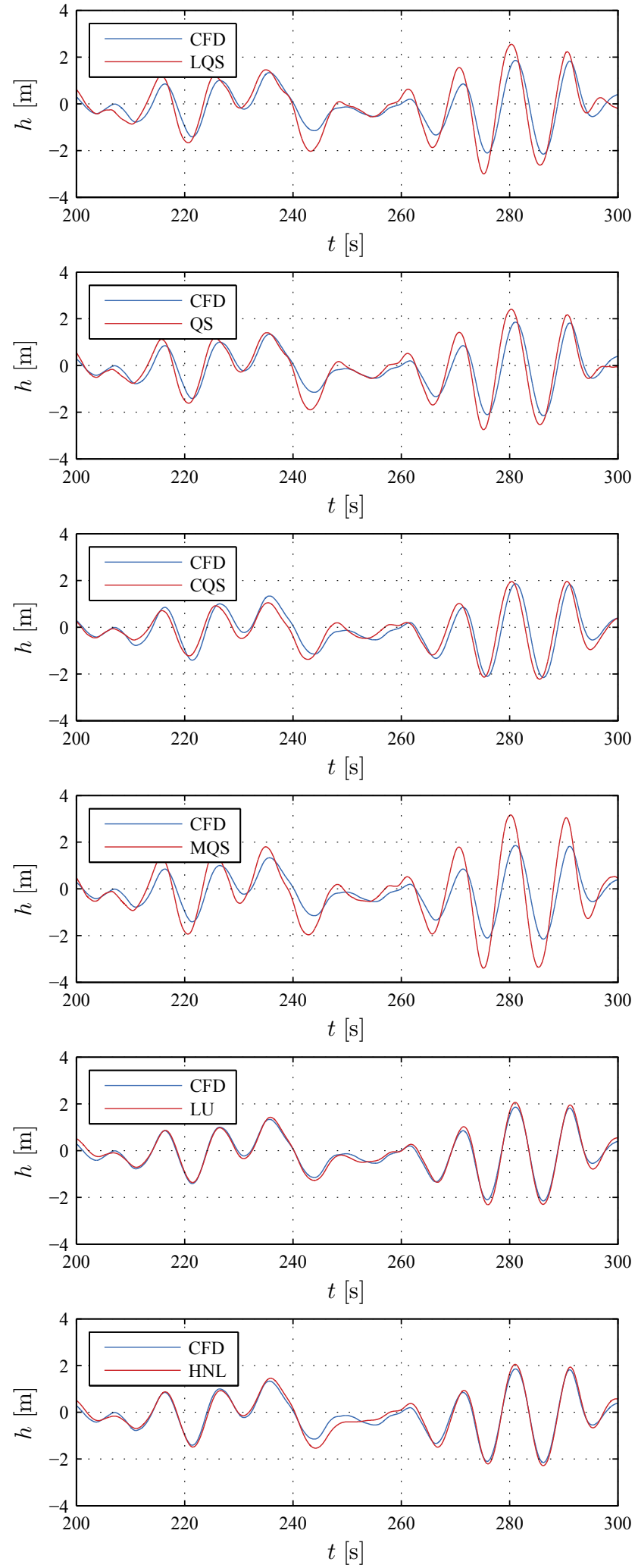
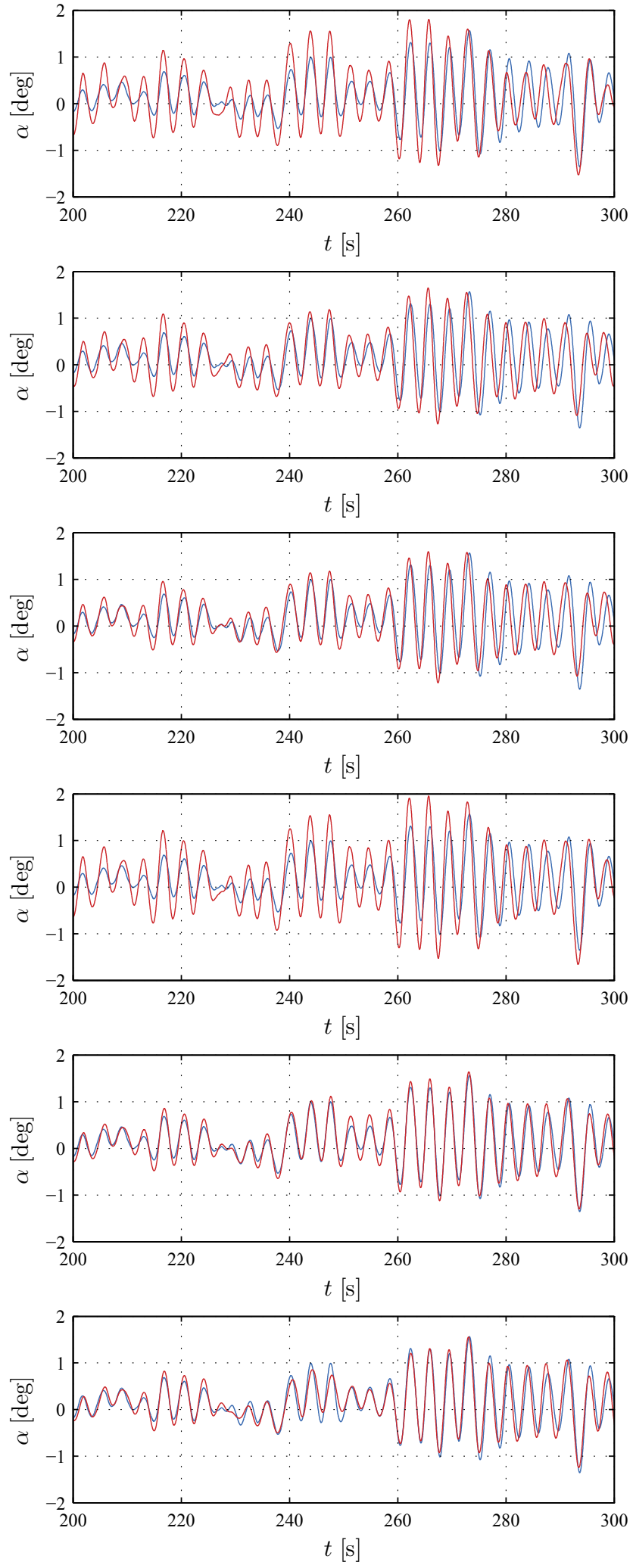

Figure 22: Representative sample time-histories of the vertical displacements $h$ (left) and rotation $\alpha$ (right) for the CFD and the semi-analytical models at $U=30 \mathrm{~m} / \mathrm{s}$ and $I=6 \%$. 

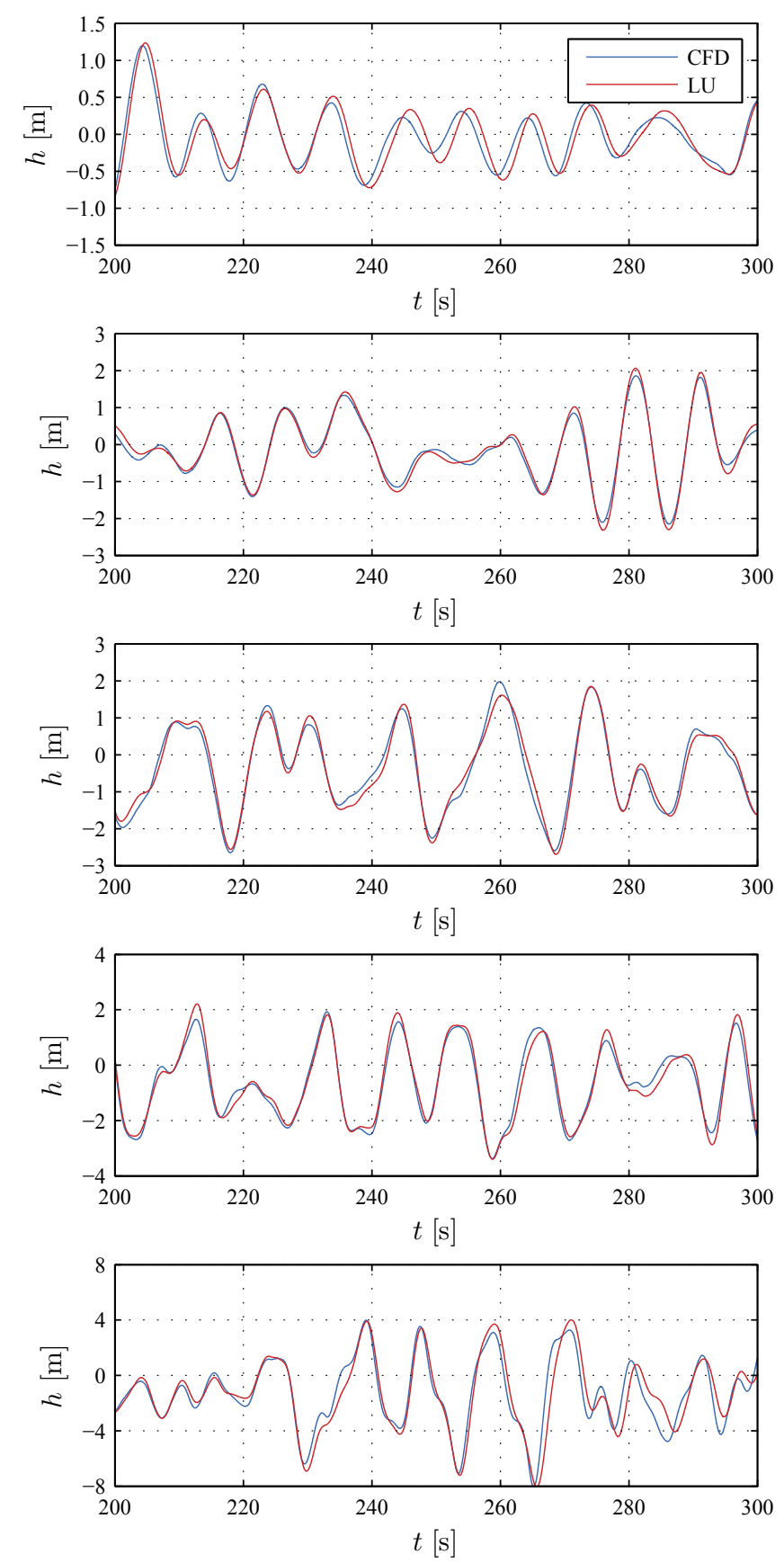
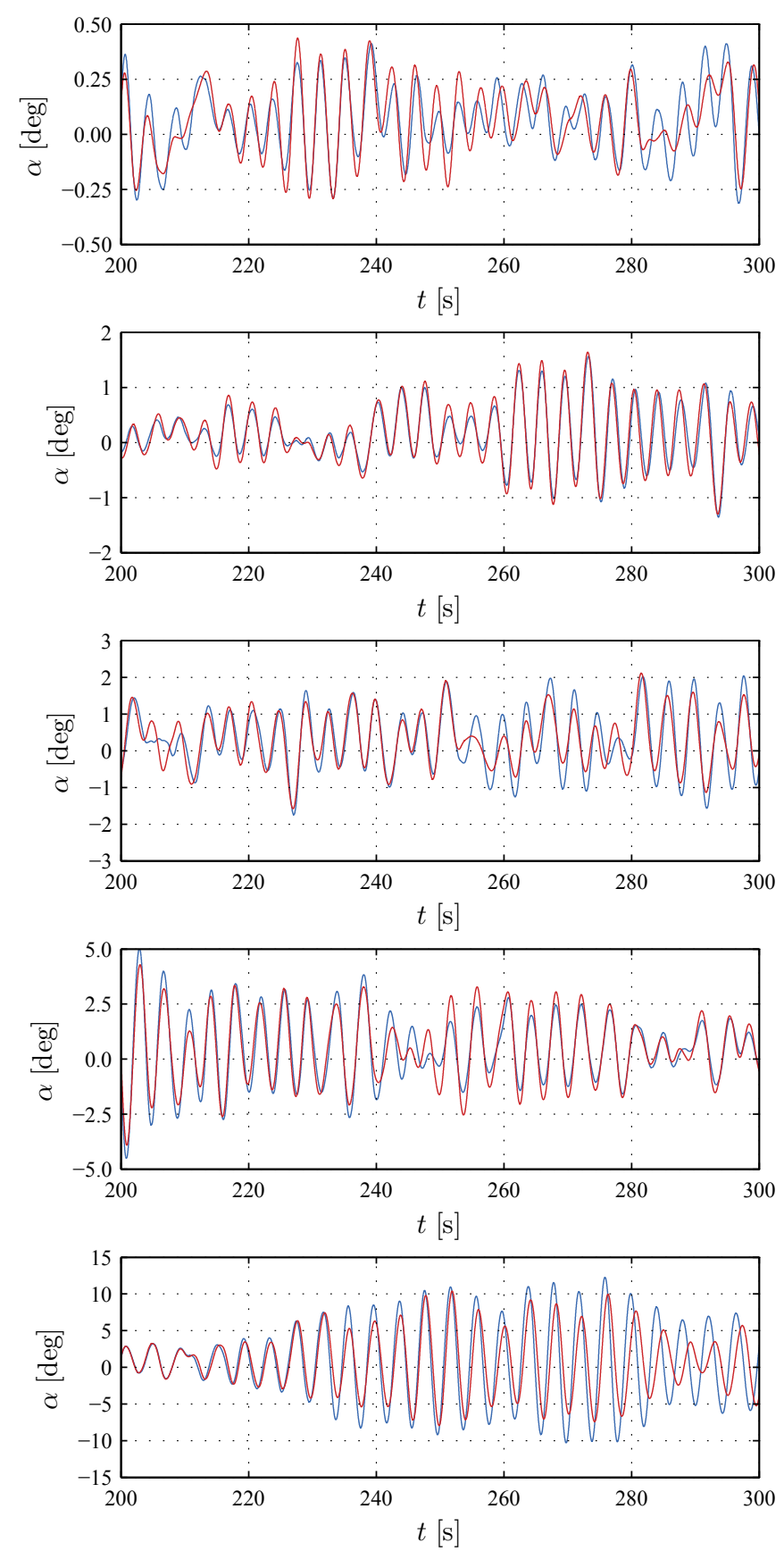

Figure 23: Representative sample time-histories of the vertical displacements $h$ (left) and rotation $\alpha$ (right) for the LU and the CFD model for $I=6 \%$. From top to bottom: $U=20,30,40,50$ and $60 \mathrm{~m} / \mathrm{s}$. 
the models. In a dynamic system, the nonlinearity of the self-excited forces is included through their nonlinear amplitude dependence on the motion (Giesing, 1968) and multi-frequency content for a single frequency motion (Diana et al., 2008, 2010). In Fig. 23, a comparison of the response time-histories for the LU and CFD models is given for the studied wind speed range and the case with low turbulence. The torsional responses for the LU and CFD models exhibit an increasing phase-shift and amplitude difference at $U=60 \mathrm{~m} / \mathrm{s}$. A fundamental study on the self-excited forces is not conducted herein. However, observations reported in former fundamental studies (Huang et al., 2014; Zhang et al., 2017) suggest that amplitude dependence of the self-excited forces and thus, complex hysteretic behavior is a plausible interpretation for the discrepancy in the torsional response for these models at high wind speed. A further indication for the nonlinearity of the self-excited forces can be realized by observing the flutter derivatives and static wind coefficients for angle $\alpha_{s} \geq 4$. The torsional response for the HNL model for the high turbulence case resulted in a better agreement with the response for the CFD model than the LU model, suggesting that the nonlinear behavior is partially accounted for in the HNL model.

Although the torsional response for the LQS model for the high turbulence case seems to be in a good agreement with the response for the CFD model, a conclusion that the LQS model describes well the aerodynamic forces is likely to be inaccurate. The good agreement is rather arbitrary as the simultaneous influence of the fading fluid memory and nonlinear effects on the aerodynamic forces cancels out for the CFD model.

\subsection{Flutter Analysis}

The critical flutter velocity $U_{c r}$ is computed in the time-domain using the semi-analytical and CFD models for smooth free-stream and the results are given in Fig. 24 (left). Additionally, the figure includes the critical flutter range obtained from direct section model tests, which is given by Larsen (1993). The critical frequency $f_{c r}$ is obtained from the FFT of the displacements at flutter (see Fig. 24, right). The good correspondence of the results for the HNL and CFD models suggests that good approximation of the critical flutter velocity can be obtained by a linear dynamic perturbation analysis about the nonlinear static equilibrium (Dowell, 2004). In case of the HNL model, the flutter derivatives were linearized at the static angle $\alpha_{s}=1.5 \mathrm{deg}$ for the flutter analysis. However, the effect of the aerostatic nonlinearity is not very significant in this case as the critical velocity for the LU model is slightly overestimated $(\approx 2.5 \%)$ with respect to the one for the HNL model. The QS and LQS models underestimate the critical velocity compared to the LU and CFD models, which indicates that the effect of linear fluid memory increases the flutter velocity. This observation is in contradiction to the results presented by $\mathrm{Wu}$ and Kareem (2013); however, a general conclusion cannot be made since the flutter derivatives and the aerodynamic center are case-dependent. Furthermore, from the results for the QS and CQS models, it can be observed that including the averaged fluid memory increases the flutter velocity when the quasi-steady nonlinearity is considered. Nevertheless, considering the fluid memory in an averaged manner overestimates the effect on the instability threshold as the critical velocity for the CQS model is overestimated compared to the one for the LU, HNL and CFD models. A higher flutter velocity is obtained for the MQS model compared to the CFD model, which is a consequence of the quadratic trend of the velocity-related flutter derivatives. Figure 25 depicts the time-histories of the decaying, critical and post-critical response for the CFD, HNL and QS models. The response of the CFD model below $U_{c r}$ has a decaying trend, while limit cycle oscillations (LCO) can be observed at $U_{c r}$. In case of the CFD free vibration analysis, it is difficult to obtain the exact critical velocity, although $U_{c r}$ was computed with an increment of $0.2 \mathrm{~m} / \mathrm{s}$. The response for the HNL and QS models maintain a constant amplitude at the critical flutter speed. For a velocity higher than $U_{c r}$, the response for the HNL model adopts divergent behavior with unlimited amplitudes. Although the post-flutter behavior results in LCO for the QS model, the amplitudes during LCO are not corresponding with the CFD model. The reason for this is that the dynamic wind coefficients considerably exceed their static values, as noted by McCroskey (1982). The aerodynamic response from the flutter analysis for the rest of the semi-analytical models is omitted herein, since it has a similar trend as the LU and QS models (linear or nonlinear) and is further discussed by Wu and Kareem (2013).

An absolute value of the wavelet coefficients $W(t, f)$ of the torsional response for the CFD model, normalized with respect to the frequency marginal, is depicted in Fig. 26 (left). For the wavelet analysis, the Morlet parent wavelet is used with a central frequency of $0.5 \mathrm{~Hz}$. Figure 26 (right) also shows the instantaneous frequency of the torsional and vertical response for the CFD model at flutter velocity. The instantaneous frequency is computed by identification of the local maxima of the ridge where the wavelet energy is concentrated (Kijewski-Correa and Kareem, 2006). The results suggest that the initial frequency of oscillation for the CFD model corresponds well with the one obtained for the HNL
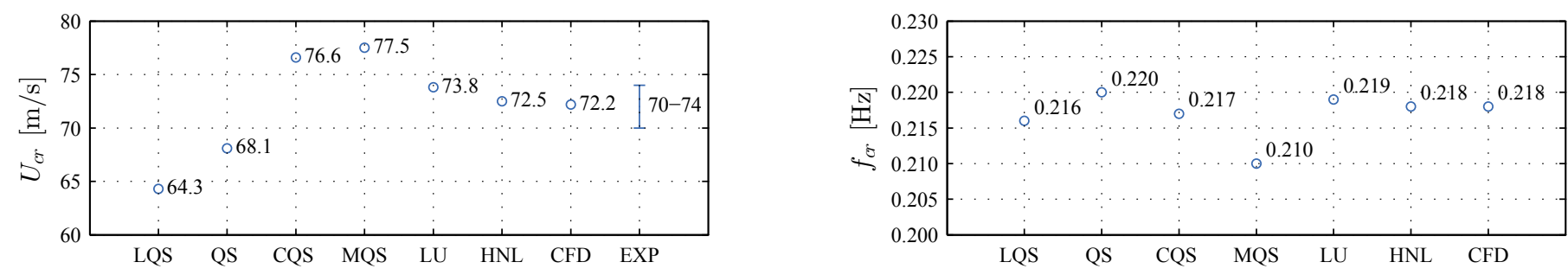

Figure 24: Critical flutter velocity $U_{c r}$ (left) and frequency $f_{c r}$ (right) for the CFD and semi-analytical models. The experimental (EXP) critical velocity is obtained as an interval from section model testing from Larsen (1993). 
a)
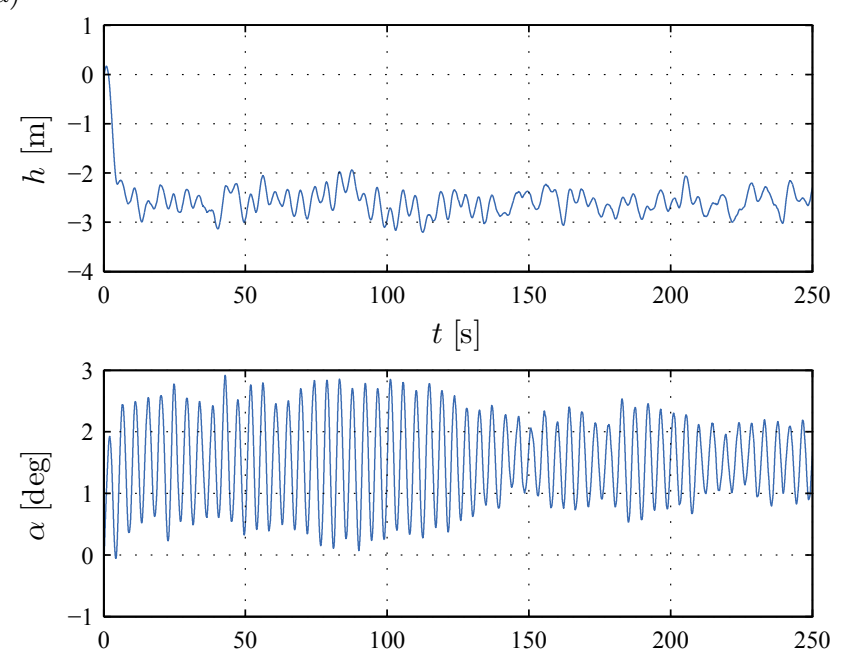

b)
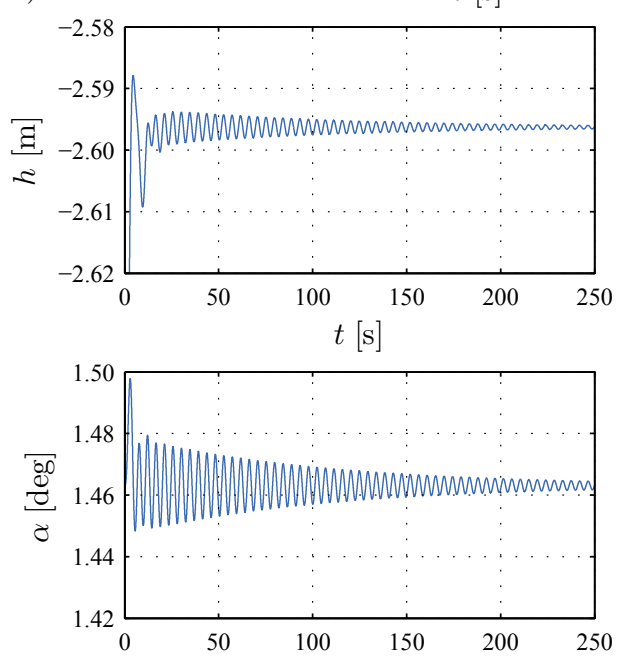

c)
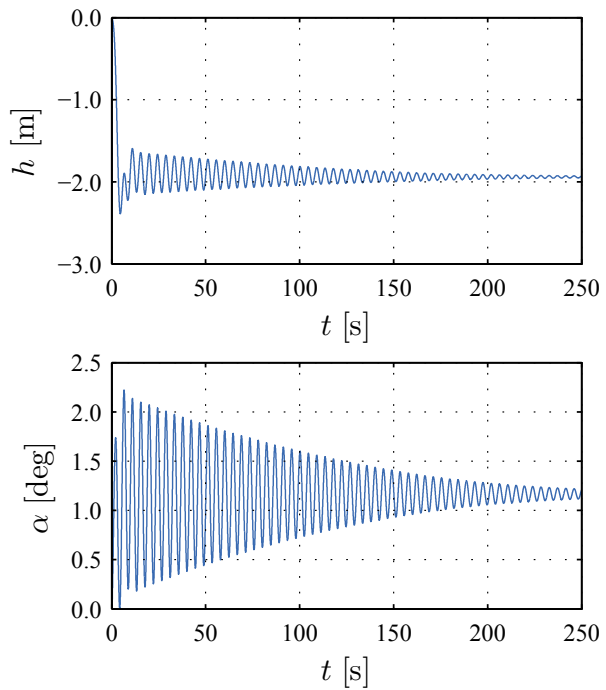
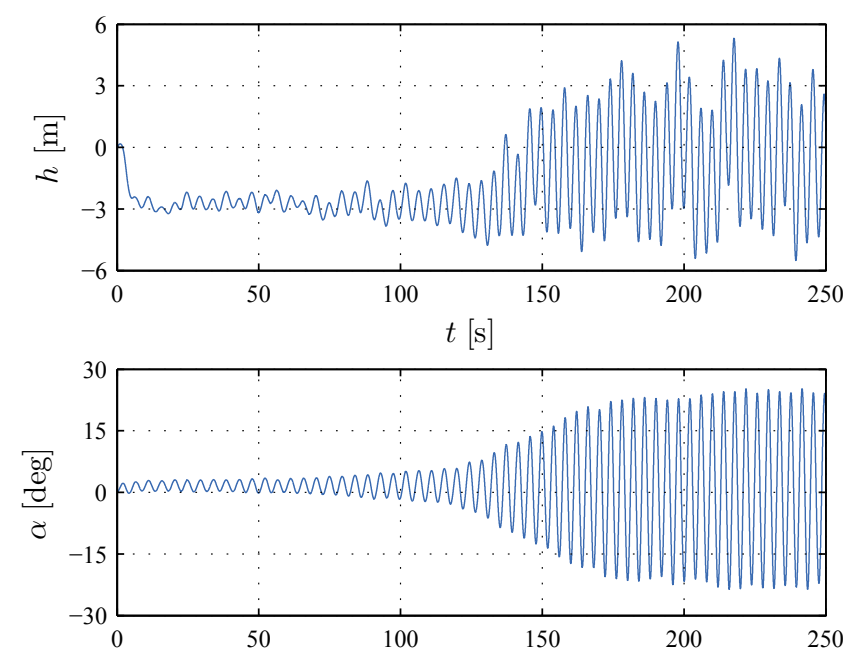

$t[\mathrm{~s}]$
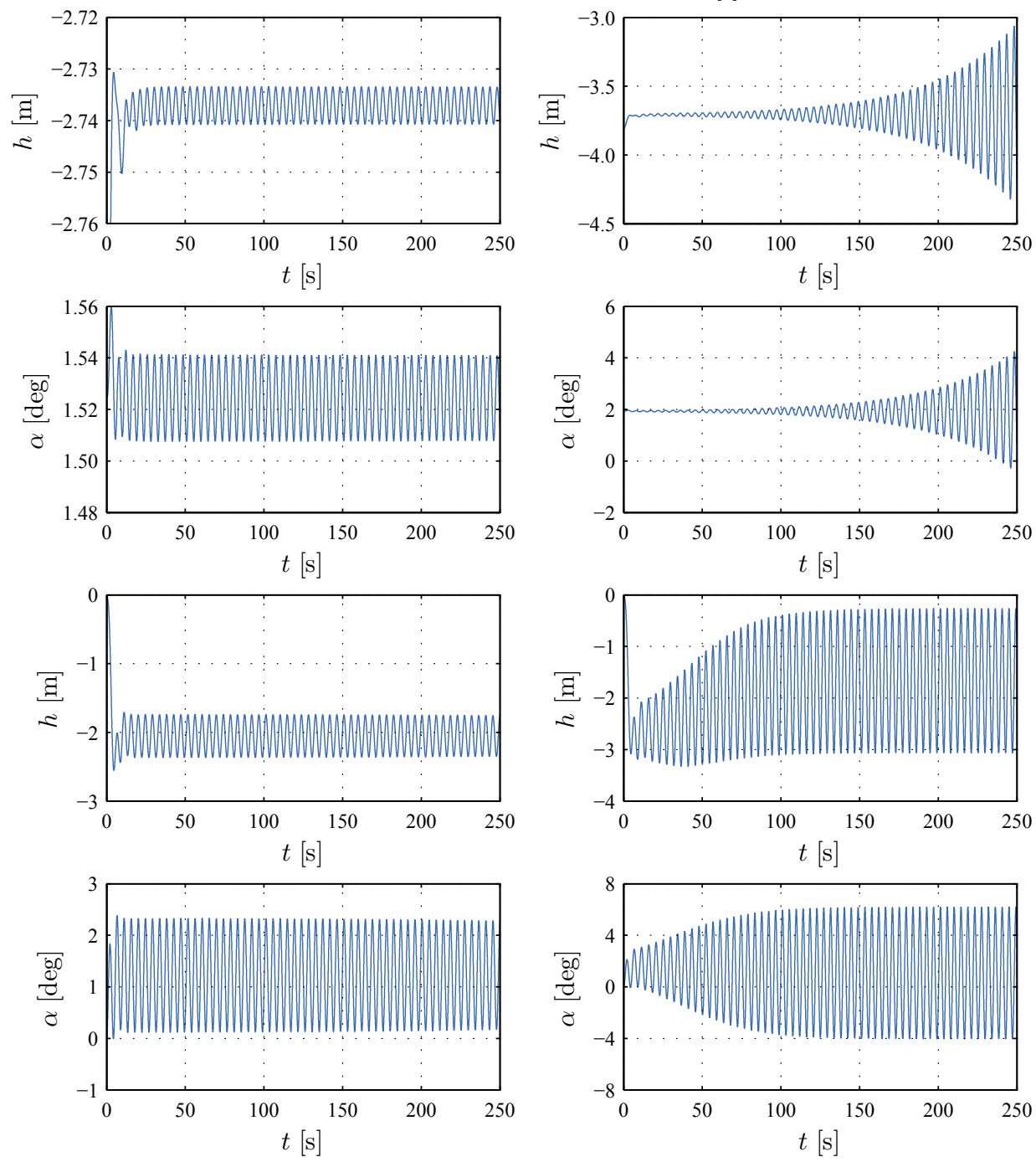

Figure 25: Time histories of vertical $h$ and torsional $\alpha$ response under smooth free-stream: a) CFD model damped response at $U=71.0 \mathrm{~m} / \mathrm{s}$ (left) and LCO at the critical flutter velocity $U=72.2 \mathrm{~m} / \mathrm{s}$ (right); b) HNL model damped response at $U=71.5 \mathrm{~m} / \mathrm{s}$ (left), critical response at $U=72.5 \mathrm{~m} / \mathrm{s}$ (center) and divergent response at $U=78.0 \mathrm{~m} / \mathrm{s}$ (right); c) QS model damped response at $U=67.0 \mathrm{~m} / \mathrm{s}$ (left), critical response at $U=68.1 \mathrm{~m} / \mathrm{s}$ (center) and LCO at $U=72.0 \mathrm{~m} / \mathrm{s}$ (right). 

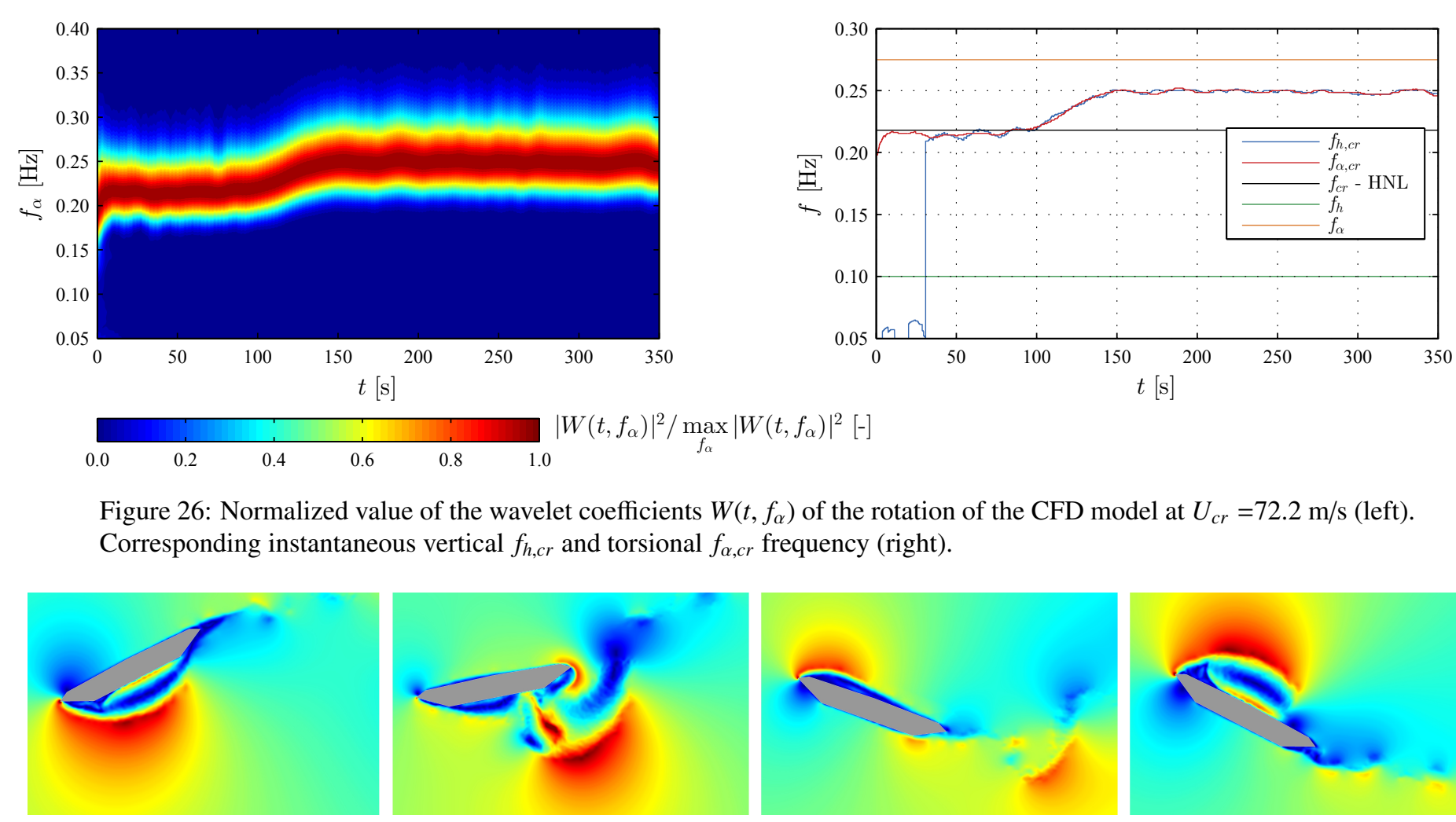

Figure 26: Normalized value of the wavelet coefficients $W\left(t, f_{\alpha}\right)$ of the rotation of the CFD model at $U_{c r}=72.2 \mathrm{~m} / \mathrm{s}$ (left). Corresponding instantaneous vertical $f_{h, c r}$ and torsional $f_{\alpha, c r}$ frequency (right).

Figure 27: Instantaneous velocity fields during LCO, from minimum (nose-down, left) up to maximum (nose-up, right) rotation.

model. As the divergent behavior progresses to stable LCO, the coupled instantaneous frequency tends to the torsional natural frequency, indicating torsionally driven oscillations in the LCO range. Amandolese et al. (2013) performed an experimental study on LCO of a flat plate due to aerodynamic nonlinearities and noted that beyond the critical velocity, the coupled frequencies tend towards the rotational branch. This is in line with the observations of this study. The authors also noted that with increasing amplitudes, there might be a change in the driving mechanism of the aeroelastic response from coupled to stall flutter. Unlike coupled flutter, stall flutter for airfoils is characterized by limited amplitudes and by partial or complete separation of the flow at the leading edge (Dowell, 2004; McCroskey, 1982), leading to high nonlinearity in the aerodynamic forces. The velocity contours during LCO, depicted in Fig. 27, indicate such separation bubble forming at the leading edge, which is characteristic for a dynamic stall. The transition from coupled to torsional flutter can be also identified from the $A_{2}^{*}$ derivative at $\alpha_{s}=4 \mathrm{deg}$ (see Sec. 4.2). Although the hysteretic behavior during dynamic stall has been studied previously using the vortex method (Akbari and Price, 2003), it is noted that amplitudes in the LCO range might lack numerical accuracy, as issues were noted in the vortex release algorithm for large rotations $\alpha>20 \mathrm{deg}$. Nevertheless, the critical flutter limit for the CFD model corresponds well with the wind tunnel experiments and the LU and HNL models, which is of main interest in bridge aerodynamics.

\section{Critical Remarks}

Three remarks are addressed in this section, regarding the strip assumption, turbulence modeling and the comparability of results from this study. First, the present investigation is based on the 2D strip assumption, which inevitably influences the fluid-structure interaction and turbulent energy transfer compared to a 3D case. The linear motion-induced forces are commonly assumed as fully correlated (Scanlan, 1997), indicating that the 2D effects are predominant for these forces. Thus, the flutter derivatives are in good correspondence with experimental results in this case. Similar observations were reported by Bai et al. (2010) for the flutter derivatives obtained using 2D and 3D numerical simulations; however, some recent experimental studies (Li et al., 2016) indicate that the $3 \mathrm{D}$ effects on the self-excited forces should be further examined. The 3D effect in the incident part of the buffeting forces is, however, not negligible and it is dependent on the free-stream turbulence characteristics such as the turbulent length scale and span-wise coherence (Larose, 2002). Herein, the employed 2D aerodynamic admittance only depends on the chord-wise wavenumber and is closely related to the complex admittance obtained from experimental tests using an active turbulence generator (Diana et al., 2002). It is well-established that the buffeting forces are dependent on the span- and chord-wise wave numbers. Experimental studies (Larose, 2002) have shown that the buffeting forces per unit span are reduced and their and span-wise correlation is increased in the 3D case. Nevertheless, a recent study by Massaro and Graham (2015) showed in analytical manner that for bridges with a large span-to-width ratio, such as the Great Belt Bridge, the strip assumption may be used with a 
sufficient accuracy as the reduction of the lift in the 3D admittance is balanced by its increased span-wise correlation. The largest influence of the three-dimensionality is probably in the local effects in the aerodynamic forces due to fluctuations with small turbulent scales and vortex shedding, as observed in experimental studies (Hiller and Cherry, 1981; Li et al., 2016).

Second, the turbulence modeling is another factor that affects the aerodynamic forces. The mechanism of energy transfer between scales for 2D turbulence is different from the mechanism for the 3D case (Boffeta and Ecke, 2012). Herein, modeling of the turbulent sub-grid scales has not been included and the resolved scales are significantly larger than the Kolmogorov length scale, hence the name CFD "model". However, it is noted that the unresolved scales do not significantly affect the resolution of the resolved scales in the vortex particle method (Cottet et al., 2002) and the angular impulse and energy are mostly conserved. Although a partial turbulence modeling is implied in the mollification process (see Sec. 3.1), this is not a controlled procedure (Cottet and Koumoutsakos, 2000). Nevertheless, the influence of turbulence, 2D or 3D, on the aerodynamic forces is not well understood and warrants further investigations, as discussed by $\mathrm{Wu}$ and Kareem (2015). In case the aerodynamic coefficients are validated to a certain extent with experimental and numerical results from former studies, it is reasonable to assume that the computational model describes the fluid-structure interaction adequately.

Third, since the aerodynamic coefficients are obtained from the CFD analyses, the CFD model is of a higher complexity than the presented 2D semi-analytical models. This means that the discussion on the underlying assumptions in the semi-analytical models is based on an one-to-one comparison. Recent aerodynamic models based on the aerodynamic hysteresis approximation (Diana et al., 2010) and Volterra series (Wu and Kareem, 2015) yielded promising results in replicating a part of the nonlinear behavior. However, they were not considered in this case and may represent a topic for further studies.

\section{Summary}

In summary, the present study investigated the wind-bridge interaction due to smooth and turbulent inflow using a CFD model, based on the vortex particle method, and six semi-analytical models. Initially, a flow verification of the synthetic free-stream turbulence was performed, concluding that the free-stream turbulence characteristics in the domain are uniform without insignificant loss of energy for isotropic turbulence. Aerodynamic coefficients including static wind coefficients, flutter derivatives and aerodynamic admittance functions were determined and compared with available numerical and experimental results of former studies. The first indications of nonlinearities were identified based on the aerodynamic coefficients for various angles of attack.

The similarities and discrepancies in the buffeting forces, aerodynamic response and critical flutter velocity for the CFD and semi-analytical models were discussed from a perspective of the inherent assumptions in the semi-analytical models in a systematic manner. Taking into consideration the buffeting forces and aeroelastic response, the LU and HNL models yielded the best correspondence with the CFD model, compared to the rest of the semi-analytical models. Thus, the fluid memory was determined to be the most influential effect in the fluid-structure interaction. However, the discrepancies between these models increased for the rotation at a particular oscillation amplitude. This was attributed to the nonlinearity of the self-excited forces, which was partially considered by the HNL model. The quasi-steady based models overestimated the effect of the nonlinearity, which was particularly observed in the PDFs of the buffeting forces and RMS of the torsional response. The aerostatic analyses demonstrated that accounting for the static nonlinearity is necessary for an adequate description of the static displacements. None of the semi-analytical models was able to replicate the effect of vortex shedding and local non-stationary effects included in the CFD model.

The corresponding critical flutter velocities obtained using the CFD and HNL models indicated that the linearized unsteady dynamic perturbation analysis at the nonlinear static angle of attack yields satisfactory results for the aerodynamic instability threshold. However, a nonlinear semi-analytical model including fluid memory is required to simulate LCO with comparable amplitudes and critical velocity with the CFD model. A change from coupled to torsional flutter mechanism was observed during the LCO. However, this requires further validation.

Finally, the notion of the study was to examine the range of applicability for the semi-analytical aerodynamic models from a CFD perspective. Although the results presented herein are for a specific case-study, it is expected that the generality extents for streamlined bridge decks. Further investigations conducted in the similar synergistic manner for bluff sections, such as multi-box girders, would additionally illuminate the suitability of the semi-analytical aerodynamic models in bridge aerodynamics. The outcome of this work is indented to shed some light on the complex wind-bridge interaction and to unveil some of the nonlinear aerodynamic effects.

\section{Acknowledgment}

This research is supported by the German Research Foundation (DFG) [Project No. 43475018], which is gratefully acknowledged by the authors. 


\section{References}

Abbas, T., Kavrakov, I., Morgenthal, G., 2017. Methods for flutter stability analysis of long-span bridges: a review. Proc Inst Civ Eng- Bridge Eng 170, 271-310. doi:10.1680/jbren.15.00039.

Akbari, M.H., Price, S.J., 2003. Simulation of dynamic stall for a NACA 0012 airfoil using a vortex method. J Fluids Struct 17 , 855-874. doi:10.1016/S0889-9746(03)00018-5.

Amandolese, X., Michelin, S., Choquel, M., 2013. Low speed flutter and limit cycle oscillations of two-degree-of-freedom flat plate in wind tunnel. J Fluids Struct 43, 244-255. doi:10.1016/j.jfluidstructs.2013.09.002.

Bai, Y., Sun, D., Lin, J., 2010. Three dimensional numerical simulations of long-span bridge aerodynamics, using block-iterative coupling and DES. Comput Fluids 39, 1549-1561. doi:10.1016/j.compfluid.2010.05.005.

Boffeta, G., Ecke, R.E., 2012. Two-dimensional turbulence. Annu Rev Fluid Mech 44, 427-451. doi:10.1146/annurev-fluid-120710-101240.

Bruno, L., Khris, S., 2003. The validity of 2D numerical simulations of vortical structures around bridge deck. Math Comput Model 37, $795-828$. doi:10.1016/S0895-7177(03)00087-6.

Brusiani, F., de Miranda, S., Patruno, L., Ubertini, F., Vaona, P., 2013. On the evaluation of bridge deck flutter derivatives using RANS. J Wind Eng Ind Aerodyn 119, 39-47. doi:10.1016/j.jweia.2013.05.002.

Caracoglia, L., Jones, N.P., 2003. Time domain vs. frequency domain characterization of aeroelastic forces for bridge deck sections. J Wind Eng Ind Aerodyn 91, 371-402. doi:10.1016/S0167-6105(02)00399-9.

Chen, X., 2006. Analysis of long span bridge response to winds: building nexus between flutter and buffeting. J Struct Eng 132, $2006-2017$. doi:10.1061/(ASCE)0733-9445(2006)132:12(2006).

Chen, X., Kareem, A., 2001. Nonlinear response analysis of long-span bridges under turbulent winds. J Wind Eng Ind Aerodyn 89, $1335-1350$. doi:10.1016/S0167-6105(01)00147-7.

Chen, X., Kareem, A., 2002. Advances in modeling of aerodynamic forces on bridge decks. J Eng Mech 128, 1193-1205. doi:10.1061/(ASCE)07339399(2002)128:11(1193).

Cherry, N.J., Hiller, R., Latour, M.E.M.P., 1984. Unsteady measurements in a separated and reattaching flow. J Fluid Mech 144 , $13-46$. doi: $10.1017 /$ S002211208400149X.

Chorin, A., 1973. Numerical study of slightly viscous flow. J Fluid Mech 27, 785-796. doi:10.1017/S0022112073002016.

Cottet, G., Koumoutsakos, P., 2000. Vortex methods: Theory and Practice. Cambridge University Press.

Cottet, G.H., Michaux, B., Ossia, S., VanderLinden, G., 2002. A comparison of spectral and vortex methods in three-dimensional incompressible flows J Compt Phys 175, 1-11. doi:10.1006/jcph.2001.6963.

Davenport, A.G., 1962. The response of slender, line-like structures to a gusty wind. Proc Inst Civ Eng 23, 389-408. doi:10.1680/iicep.1962.10876.

Deodatis, G., 1996. Simulation of ergodic multivariate stochastic processes. J Eng Mech 122, 778-787. doi:10.1061/(ASCE)07339399(1996)122:8(778).

Diana, G., Bruni, S., Cigada, A., Collina, A., 1993. Turbulence effect on flutter velocity in long span suspended bridges. J Wind Eng Ind Aerodyn 48, 329-342. doi:10.1016/0167-6105(93)90144-D.

Diana, G., Bruni, S., Cigada, A., Zappa, E., 2002. Complex aerodynamic admittance function role in buffeting response of a bridge deck. J Wind Eng Ind Aerodyn 90, 2057-2072. doi:10.1016/S0167-6105(02)00321-5.

Diana, G., Resta, F., Rocchi, D., 2008. A new numerical approach to reproduce bridge aerodynamic non-linearities in the time domain. J Wind Eng Ind Aerodyn 96, 1871-1884. doi:10.1016/j.jweia.2008.02.052.

Diana, G., Rocchi, D., Argentini, T., 2013. An experimental validation of band superposition model of the aerodynamic forces acting on a multi-box deck sections. J Wind Eng Ind Aerodyn 113, 40-58. doi:10.1016/j.jweia.2012.12.005.

Diana, G., Rocchi, D., Argentini, T., Muggiasca, S., 2010. Aerodynamic instability of a bridge deck section model: Linear and nonlinear approach to force modeling. J Wind Eng Ind Aerodyn 98, 363-374. doi:j.jweia.2010.01.003.

Ding, Q., Zhu, L., Xiang, H., 2011. An efficient ergodic simulation of multivariate stochastic process with spectral representation. Prob Eng Mech 26, 350-356. doi:10.1016/j.probengmech.2010.09.006.

Dowell, E., 2004. A modern course in aeroelasticity. Fourth ed., Kluwer Academic Publishers.

Farsani, H.Y., Valentine, D.T., Arena, A., Lacarbonara, W., Marzocca, P., 2014. Indicial functions in the aeroelasticity of bridge decks. J Bridge Eng 19, 1-13. doi:10.1016/j.jfluidstructs.2014.02.015.

Ferziger, J., 1993. Simulation of complex turbulent flows: recent advances and prospects in wind engineering. J Wind Eng Ind Aerodyn 46-67, $195-212$. doi:10.1016/0167-6105(93)90130-G.

Frandsen, J.B., 2004. Numerical bridge deck studies using finite elements. Part I: Flutter. J Fluids Struct 19, 171-191. doi:10.1016/j.jfluidstructs.2003.12.005.

Fukuda, K., Kamemoto, K., 2004. A grid-free redistribution model for a vortex method and turbulent flow analysis. Kyoto, Japan. pp. $183-188$.

Fung, Y., 1993. An introduction to the theory of aeroelasticity. Dover Edition ed., Dover Publications, Inc., New York.

Ge, Y., Xiang, H.F., 2008. Computational models and methods for aerodynamic flutter of long-span bridges. J Wind Eng Ind Aerodyn 96, $1912-1924$. doi:10.1016/j.jweia.2008.02.017.

Giesing, J.P., 1968. Nonlinear two-dimensional unsteady potential flow with lift. J Aircr 5, 135-143. doi:10.2514/3.43920.

Haan, F.L., Kareem, A., 2016. Anatomy of turbulence effect on aerodynamics of an oscillating prism. J Eng Mech 135, 987-999. doi:10.1061/(ASCE)EM.1943-7889.0000012.

Hejlesen, M.M., Rasmussen, J.T., Larsen, A., Walther, J.H., 2015. On estimating aerodynamic admittance of bridge section by mesh-free vortex method. J Wind Eng Ind Aerodyn 146, 117-127. doi:10.1016/j.jweia.2015.08.003.

Hiller, R., Cherry, N.J., 1981. The effects of stream turbulence on separation bubbles. J. Wind Eng Ind Aerodyn 8, 49-58. doi:10.1016/01676105(81)90007-6.

Hockney, R., Eastwood, J., 1988. Computer Simulation Using Particles. Taylor \& Francis.

Huang, L., Xu, Y.L., Liao, H., 2014. Nonlinear aerodynamic forces on thin flat plate: Numerical study. J Fluids Struct 44, 182-194. doi:10.1016/j.jfluidstructs.2013.10.009.

Jain, A., Jones, N.P., Scanlan, R.H., 1996. Coupled flutter and buffeting analysis of long-span bridges. J Struct Eng 122 , 716-725. doi:10.1061/(ASCE)0733-9445(1996)122:7(716).

Kareem, A., Wu, T., 2013. Wind-induced effects on bluff bodies in turbulent flows: nonstationary, non-Gaussian and nonlinear features. J Wind Eng Ind Aerodyn 122, 21-37. doi:10.1016/j.jweia.2013.06.002.

Kavrakov, I., Morgenthal, G., 2017. A comparative assessment of aerodynamic models for buffeting and flutter of long-span bridge. Eng 3, 823-838. doi:10.1016/j.eng.2017.11.008

Kijewski-Correa, T., Kareem, A., 2006. Efficiency of Hilbert and wavelet transforms for time-frequency analysis. J Eng Mech 132, $1037-1049$. doi:10.1061/(ASCE)0733-9399(2006)132:10(1037).

Kovacs, I., Svensson, H.S., Jordet, E., 1992. Analytical aerodynamic investigation of cable-stayed Helgeland bridge. J Struct Eng 118, $147-168$. doi:10.1061/(ASCE)0733-9445(1992)118:1(147)

Kristensen, L., 1979. On longitudinal spectral coherence. Bound-Lay Meteorol 16, 145-153. doi:10.1007/BF02350508. 
Kuroda, S., 1997. Numerical simulation of flow around a box girder of a long span suspension bridge. J Wind Eng Ind Aerodyn 67 \& 68, $239-252$. doi:10.1016/S0167-6105(97)00076-7.

Larose, L., 2002. The dynamic action of gusty winds on long-span bridges. Technical Report BYG-DTU R-029.

Larsen, A., 1993. Aerodynamic aspects of the final design of the 1624m suspension bridge across the Great Belt. J Wind Eng Ind Aerodyn 48, $261-285$. doi:10.1016/0167-6105(93)90141-A.

Larsen, A., Walther, J.H., 1997. Aeroelastic analysis of bridge girder sections based on discrete vortex simulations. J Wind Eng Ind Aerodyn 67\&68, 253-265. doi:10.1016/S0167-6105(97)00077-9.

Lee, N., Lee, H., Baek, C., Lee, S., 2016. Aeroelastic of bridge deck flutter with modified implicit coupling method. J Wind Eng Ind Aerodyn 155, 11-22. doi:10.1016/j.jweia.2016.04.010.

Li, M., Li, S., Liao, H., Zeng, J., Wang, Q., 2016. Spanwise correlation of aerodynamic forces on oscillating rectangular cylinder. J Wind Eng Ind Aerody 154, 47-57. doi:10.1016/j.jweia.2016.04.003.

Mann, J., 1994. The spatial structure of neutral atmospheric surface-layer turbulence. J Fluid Mech 273, 141-168. doi:10.1017/S0022112094001886.

Massaro, M., Graham, J., 2015. The effect of three-dimensionality on the aerodynamic admittance of thin sections in free stream turbulence. J Fluids Struct 57, 81-90. doi:10.1016/j.jfluidstructs.2015.05.012.

McCroskey, W.J., 1982. Unsteady airfoils. Ann Rev Fluid Mech 14, 285-311. doi:10.1146/annurev.fl.14.010182.001441.

Morgenthal, G., 2002. Aerodynamic analysis of structures using high-resolution vortex particle methods. Ph.D. thesis. Cambridge University.

Morgenthal, G., Corriols, A., Bendig, B., 2014. A GPU-accelerated pseudo-3D vortex method for aerodynamic analysis. J Wind Eng Ind Aerodyn 125, 69-80. doi:10.1016/j.jweia.2013.12.002

Morgenthal, G., McRobie, F.A., 2002. A comparative study of numerical methods for fluid-structure interaction analysis in long-span bridge design. Wind Struct 5, 101-114. doi:10.12989/was.2002.5.2_3_4.101.

Morgenthal, G., Walther, J., 2007. An immersed interface method for the Vortex-In-Cell algorithm. Comput Struct 85, 712-726. doi:10.1016/j.compstruc.2007.01.020.

Øiseth, O., Rönnquist, A., Sigbjörnsson, 2010. Simplified prediction of wind-induced response and stability limit of slender long-span suspension bridges, based on modified quasi-steady theory: A case study. J Wind Eng Ind Aerodyn 98, 730-741. doi:10.1016/j.jweia.2010.06.009.

Prendergast, J., 2007. Simulation of 2D unsteady wind by a vortex method. Ph.D. thesis. Cambridge University.

Prendergast, J., McRobie, F.A., 2006. Simulation of 2D unsteady wind by a vortex method and application to studying bluff body flow, in: 7th UK Conf Wind Eng.

Rasmussen, J.T., Hejlesen, M.M., Larsen, A., Walther, J.H., 2010. Discrete vortex method simulations of the aerodynamic admittance in bridge aerodynamics. J Wind Eng Ind Aerodyn 99, 776-785. doi:10.1016/j.jweia.2010.06.011.

Reinhold, T., Brinch, M., Damsgaard, A., 1992. Wind tunnel tests for the Great Belt Link, in: Larsen, A. (Ed.), Aerodynamics of Large Bridges, Copenhagen, Denmark. pp. 255-267.

Saathof, P.J., Melbourne, W.H., 1997. Effects of free-stream turbulence on surface pressure fluctuations in a separation bubble. J Fluid Mech 338, 1-24. doi:10.1017/S0022112096004594.

Scanlan, R.H., 1978. The action of flexible bridges under wind, I: Flutter theory. J Sound Vib 60, 187-199. doi:10.1016/S0022-460X(78)80028-5.

Scanlan, R.H., 1997. Amplitude and turbulence effects on bridge flutter derivatives. J Struct Eng 123, 232-236. doi:10.1061/(ASCE)07339445(1997)123:2(232).

Scanlan, R.H., 2000. Motion-related body-force functions in two-dimensional low-speed flow. J Fluids Struct 14, 49-63. doi:10.1006/jfls.1999.0252.

Scanlan, R.H., Béliveau, J.G., Budlong, K.S., 1974. Indicial aerodynamic functions for bridge decks. J Eng Mech 100, 657-672.

Sears, W.R., 1941. Some aspects of non-stationary airfoil theory and its practical application. J AIAA 8, 104-108. doi:10.2514/8.10655.

Shinozuka, M., Deodatis, G., 1996. Simulation of multi-dimensional Gaussian stochastic fields by spectral representation. Appl Mech Rev 49 , $29-53$. doi: $10.1115 / 1.3101883$.

Simiu, E., Scanlan, R., 1996. Wind effects on structures. Third ed., Jon Wiley \& Sons, INC.

Solari, G., Piccardo, G., 2000. Probabilistic 3-D turbulence modeling for gust buffeting of structures. Prob Eng Mech 16, 73-86. doi:10.1016/S02668920(00)00010-2

Taylor, I.J., Vezza, M., 2009. A numerical investigation into the aerodynamic characteristics and aeroelastic stability of a footbridge. J Fluids Struct 144, 155-177. doi:10.1016/j.jfluidstructs.2008.05.001.

Theodorsen, T., 1949. General theory of aerodynamic instability and the mechanism of flutter. Technical Report 496. NASA. Washington, USA.

Vairo, G., 2003. A numerical model for wind loads simulation on long-span bridges. Simul Model Pract Theory 11, 315-351. doi:10.1016/S1569$190 X(03) 00053-4$.

Wu, J.C., 1976. Numerical boundary conditions for viscous flow problems. AIAA 14, 1042-1049. doi:10.2514/3.61439.

Wu, T., Kareem, A., 2013. Bridge aerodynamics and aeroelasticity: A comparison of modeling schemes. J Fluids Struct 43, 347-370. doi:10.1016/j.jfluidstructs.2013.09.015.

Wu, T., Kareem, A., 2015. A nonlinear analysis framework for bluff-body aerodynamics: A Volterra representation of the solution of Navier-Stokes equations. J Fluids Struct 54, 479-502. doi:10.1016/j.jfluidstructs.2014.12.005.

$\mathrm{Wu}, \mathrm{T} ., \mathrm{Kareem}, \mathrm{A} ., \mathrm{Ge}, \mathrm{Y}$, , 2013. Linear and nonlinear aeroelastic analysis framework for cable supported bridges. Nonlinear Dyn 74, 487-516. doi:10.1007/s11071-013-0984-7.

Zhang, X., Xiang, H., Sun, B., 2002. Nonlinear aerostatic and aerodynamic analysis of long-span suspension bridges considering wind-structure interactions. J Wind Eng Ind Aerodyn 90, 1065-1080. doi:10.1016/S0167-6105(02)00251-9.

Zhang, Z., Chen, Z., Cai, Y., Ge, Y., 2011. Indicial functions for bridge aeroelastic forces and time-domain flutter analysis. J Bridge Eng $16,546-557$. doi:10.1061/(ASCE)BE.1943-5592.0000176.

Zhang, Z., Zhang, X., Yang, Y., Ge, Y., 2017. Nonlinear aerodynamic and energy input properties of a twin-box girder deck section. J Fluids Struct 74, 413-426. doi:j.jfluidstructs.2017.06.016. 